\title{
Generalized Solutions of Functional Differential Inclusions
}

\author{
Anna Machina, ${ }^{1,2}$ Aleksander Bulgakov ${ }^{3}$ and Anna Grigorenko ${ }^{4}$ \\ ${ }^{1}$ Center for Integrative Genetics (CIGENE), Norwegian University of Life Sciences, 1432 Aas, Norway \\ ${ }^{2}$ Department of Mathematical Sciences and Technology, Norwegian University of Life Sciences, \\ 1432 Aas, Norway \\ ${ }^{3}$ Department of Algebra and Geometry, Tambov State University, 392000 Tambov, Russia \\ ${ }^{4}$ Department of Higher Mathematics, Faculty of Electronics and Computer Sciences, \\ Moscow State Forest University, 141005 Moscow, Russia
}

Correspondence should be addressed to Anna Machina, annama@umb.no

Received 12 March 2007; Revised 4 July 2007; Accepted 12 September 2007

Recommended by Yong Zhou

We consider the initial value problem for a functional differential inclusion with a Volterra multivalued mapping that is not necessarily decomposable in $L_{1}^{n}[a, b]$. The concept of the decomposable hull of a set is introduced. Using this concept, we define a generalized solution of such a problem and study its properties. We have proven that standard results on local existence and continuation of a generalized solution remain true. The question on the estimation of a generalized solution with respect to a given absolutely continuous function is studied. The density principle is proven for the generalized solutions. Asymptotic properties of the set of generalized approximate solutions are studied.

Copyright (C) 2008 Anna Machina et al. This is an open access article distributed under the Creative Commons Attribution License, which permits unrestricted use, distribution, and reproduction in any medium, provided the original work is properly cited.

\section{Introduction}

During the last years, mathematicians have been intensively studying (see [1, 2]) perturbed inclusions that are generated by the algebraic sum of the values of two multivalued mappings, one of which is decomposable. Many types of differential inclusions can be represented in this form (ordinary differential, functional differential, etc.). In the above-mentioned papers, the authors investigated the solvability problem for such inclusions. Estimates for the solutions were obtained similar to the estimates, which had been obtained by Filippov for ordinary differential inclusions (see $[3,4]$ ). The concept of quasisolutions is introduced and studied. The density principle and the "bang-bang" principle are proven. In papers [5-8], the perturbed inclusions with internal and external perturbations are considered, and the conjecture that "small" internal and external perturbations can significantly 
change the solution set of the perturbed inclusion is proven. Let us remark that, in the cited papers, the proofs of the obtained results essentially depend on the assumption that the multivalued mapping, which generates the algebraic sum of the values, is decomposable. Therefore, these studies once again confirm V. M. Tikhomirov's conjecture that decomposability is a specific feature of the space $L_{1}^{n}[a, b]$ and plays the same role as the concept of convexity in Banach spaces. The decomposability is implicitly used in many fields of mathematics: optimization theory, differential inclusions theory, and so forth. If a multivalued mapping is not necessarily decomposable, then the methods known for multivalued mappings cannot even be applied to the solvability problem of the perturbed inclusion. Furthermore, in this case, the equality between the set of quasisolutions of the perturbed inclusion and the solution set of the perturbed inclusion with the decomposable hull of the right-hand side fails. This equality for the ordinary differential inclusions was proven by Ważewski (see [9]). The point is that, in this case, the closure (in the weak topology of $\left.L_{1}^{n}[a, b]\right)$ of the set of the values of this multivalued mapping does not coincide with the closed convex hull of this set. As a result, we have that fundamental properties of the solution sets (the density principle and "bang-bang" principle) do not hold any more (see $[3,10-13])$. The situation cannot be improved even if the mapping in question is continuous.

In this paper, we consider the initial value problem for a functional differential inclusion with a multivalued mapping. We assume that this mapping is not necessarily decomposable. Some mathematical models can naturally be described by such an inclusion. For instance, so do certain mathematical models of sophisticated multicomponent systems of automatic control (see [14]), where, due to the failure of some devices, objects are controlled by different control laws (different right-hand sides) with the diverse sets of the control admissible values. This means that the object's control law consists of a set of the controlling subsystems. These subsystems may be linear as well as nonlinear. For example, this occurs in the control theory of the hybrid systems (see [15-20]). Due to the failure of a device, the control object switches from one control law to another. The control of an object must be guaranteed in spite of the fact that failures (switchings) may take place any time. Therefore, the mathematical model should treat all available trajectories (states) corresponding to all switchings. The generalized solutions of the inclusion make up the set of all such trajectories. The concept of a generalized solution should be then introduced and its properties should be studied.

We consider a functional differential inclusion with a Volterra-Tikhonov type (in the sequel simply Volterra type) multivalued mapping and we prove that for such an inclusion, the theorem on existence and continuation of a local generalized solution holds true. This justifies one of the requirements, which were formulated in the monograph of Filippov [4] for generalized solutions of differential equations with discontinuous right-hand sides. In the present paper, it is also proven that in the regular case, that is, when a multivalued mapping is decomposable, a generalized solution coincides with an ordinary solution. At the same time, the concept of a generalized solution discussed in the present paper does not satisfy all the requirements that are usually put on generalized (in the sense of the monograph [4]) solutions of differential equations with discontinuous right-hand sides. For instance, the limit of generalized (in the sense of the present paper) solutions is not necessarily a generalized solution itself. The reason for that is that a multivalued mapping that determines a generalized solution (the definition is given below) may not be closed in the weak topology of $\mathbf{L}_{1}^{n}[a, b]$, as this mapping is not necessarily convex-valued. 


\section{Preliminaries}

We start with the notation and some definitions. Let $X$ be a normed space with the norm $\|\cdot\|_{X}$. Let $B_{X}[x, \varepsilon]$ be the closed ball in the space $X$ with the center at $x \in X$ and of radius $\varepsilon>0$; if $\varepsilon=0$, then $B_{X}[x, 0] \equiv x$. Let $U \subset X$. Then $\bar{U}$ is the closure of $U$, co $U$ is the convex hull of $U$; $\overline{\operatorname{co}} U \equiv \overline{\operatorname{co} U}$, ext $U$ is the set of all extreme points of $U$; $\overline{\operatorname{ext}} U=\overline{\operatorname{ext} U}$. Let $\|U\|_{X}=\sup _{u \in U}\|u\|_{X}$. Let $U^{\varepsilon} \equiv \overline{\bigcup_{u \in U} B[u, \varepsilon]}$ if $\varepsilon>0$ and $U^{0} \equiv \bar{U}$.

Let $\rho_{X}[x ; U]$ be the distance from the point $x \in X$ to the set $U$ in the space $X$; let $h_{X}^{+}\left[U_{1} ; U\right] \equiv \sup _{x \in U_{1}} \rho_{X}[x, U]$ be the Hausdorff semideviation of the set $U_{1}$ from the set $U$; let $h_{X}\left[U_{1} ; U\right]=\max \left\{h_{X}^{+}\left[U_{1} ; U\right] ; h_{X}^{+}\left[U ; U_{1}\right]\right\}$ be the Hausdorff distance between the subsets $U_{1}$ and $U$ of $X$.

We denote by comp $[X]$ (resp., comp $\left[X^{*}\right]$ ) the set of all nonempty compact subsets of $X$ (resp., the set of all nonempty, bounded, closed in the space $X$, and relatively compact in the weak topology on the space $X$ subsets of $X$ ). Let $2^{X}$ be the set of all nonempty bounded subsets of $X$.

Let $P$ be a system of subsets of $X$ (a subset of $X$ ). We denote by $\Omega(D)$ the set of all nonempty convex subsets of $X$, belonging to the system $P$ (the set of all nonempty convex subsets of $X$, belonging to $P$ ).

Let $\mathbb{R}^{n}$ be the space of all $n$-dimensional column vectors with the norm $|\cdot|$. We denote by $C^{n}[a, b]$ (resp., $D^{n}[a, b]$ ) the space of continuous (resp., absolutely continuous) functions $x:[a, b] \rightarrow \mathbb{R}^{n}$ with norm $\|x\|_{C^{n}[a, b]}=\max \{|x(t)|: t \in[a, b]\}$ (resp., $\|x\|_{D^{n}[a, b]}=|x(a)|+$ $\int_{a}^{b}|\dot{x}(s)| d s$ ). Let $u \subset[a, b]$ be a measurable set $\mu(\mathcal{U})>0$ ( $\mu$-the Lebesgue measure). We denote by $L_{p}^{n}(\mathcal{U})$ the space of all functions $x: \mathfrak{U} \rightarrow \mathbb{R}^{n}$ such that $(x(s))^{p}$ is integrable (if $p<\infty$ ) and the space of all measurable, essentially bounded (if $p=\infty$ ) functions $x: \mathcal{U} \rightarrow \mathbb{R}^{n}$ with the norms

$$
\|x\|_{L_{p}^{n}(\mathcal{U})}=\left(\int_{\mathcal{U}}|x(s)|^{p} d s\right)^{1 / p}, \quad\|x\|_{L_{\infty}^{n}(\mathcal{U})}=\underset{s \in \mathcal{U}}{\operatorname{vraisup}}|x(s)|,
$$

respectively.

Let $\Phi \subset L_{1}^{n}[a, b]$. The set $\Phi$ is called integrally bounded if there exists a function $\varphi_{\Phi} \in$ $\mathbf{L}_{1}^{1}[a, b]$ such that $|x(t)| \leq \varphi_{\Phi}(t)$ for each $x \in \Phi$ and almost all $t \in[a, b]$. The set $\Phi$ is said to be decomposable if for each $x, y \in \Phi$ and every measurable set $\mathcal{U} \subset[a, b]$ the inclusion $\chi(\mathcal{U}) x+$ $x([a, b] \backslash \mathfrak{U}) y \in \Phi$ holds, where $x(V)$ is the characteristic function of the set $V$. We denote by $Q\left[\mathbf{L}_{1}^{n}[a, b]\right]$ (resp., $\Pi\left[L_{1}^{n}[a, b]\right]$ ) the set of all nonempty, closed, and integrally bounded (resp., nonempty, bounded, closed, and decomposable) subsets of the space $L_{1}^{n}[a, b]$.

Let $F:[a, b] \rightarrow \operatorname{comp}\left[\mathbb{R}^{n}\right]$ be a measurable mapping. Then by definition, $S(F)=$ $\left\{y \in L_{1}^{n}[a, b]: y(t) \in F(t)\right.$ for almost all $\left.t \in[a, b]\right\}$. By $C_{+}^{1}[a, b]$ (resp., $L_{+}^{1}[a, b]$ ), denote the cone of all nonnegative functions of the space $C_{1}^{1}[a, b]$ (resp., $\left.L_{1}^{1}[a, b]\right)$.

Let $f: P \rightarrow Q$ be a mapping between two partially ordered sets $P$ and $Q$ (the partial order of both sets is denoted by $\leq$ ). The mapping $f$ is isotonic if $f(x) \leq f(y)$, whenever $x \leq y$.

In this paper, the expression "measurability of a single-valued function" is always used in the sense of Lebesgue measurability and "measurability of a multivalued function" in the sense of [21]. Let $(T, \Sigma, \mu)$ be a space with finite positive measure and let $F$ be a multivalued mapping from $T$ to $\mathbb{R}^{n}$. A set $\left\{x_{v}(\cdot)\right\}\left(v \in \mathbb{N}\right.$ ) of measurable mappings from $T$ to $\mathbb{R}^{n}$ is said to approximate the multivalued mapping $F$ if the set $\left\{t \in T \mid x_{v}(t) \in F(t)\right\}$ is measurable for any $v \in \mathbb{N}$, and the set $F(t)$ belongs to the closure of its intersection with the set $\bigcup_{v \in \mathbb{N}}\left\{x_{v}(t)\right\}$ for 
almost all $t \in T$. A multivalued mapping $F$ from $T$ to $\mathbb{R}^{n}$ is called measurable if there exists a countable set of measurable mappings from $T$ to $\mathbb{R}^{n}$ that approximates the mapping $F$.

Further, let us introduce the main characteristic properties of a set that is decomposable.

Lemma 2.1. Let $\Phi \in \Pi\left[L_{1}^{n}[a, b]\right]$. Then there exists a function $u \in L_{1}^{1}[a, b]$ such that $|\varphi(t)| \leqslant u(t)$ for each function $\varphi \in \Phi$ and almost all $t \in[a, b]$.

Proof. Let $\varphi_{i} \in \Phi, i=1,2, \ldots$, be a sequence of functions such that

$$
\lim _{i \rightarrow \infty}\left\|\varphi_{i}\right\|_{L_{1}^{n}[a, b]}=\|\Phi\|_{L_{1}^{n}[a, b]} .
$$

Let us show that there exists a sequence of functions $\tilde{\varphi}_{i} \in \Phi, i=1,2, \ldots$, such that the equality (2.2) holds and

$$
\left|\tilde{\varphi}_{1}(t)\right| \leqslant\left|\tilde{\varphi}_{2}(t)\right| \leqslant\left|\tilde{\varphi}_{3}(t)\right| \leqslant \cdots \leqslant\left|\tilde{\varphi}_{i}(t)\right| \leqslant\left|\tilde{\varphi}_{i+1}(t)\right| \leqslant \cdots
$$

for almost all $t \in[a, b]$.

Indeed, let $\widetilde{\varphi}_{1}=\varphi_{1}$ and $\tilde{\varphi}_{i+1}=\chi\left(\mathcal{U}_{i}\right) \tilde{\varphi}_{i}+\chi\left(\left[a, b \backslash \mathcal{U}_{i}\right) \varphi_{i+1}, i=1,2, \ldots\right.$, where $\mathcal{U}_{i}=\{t \in$ $\left.[a, b]:\left|\tilde{\varphi}_{i}(t)\right| \geqslant\left|\varphi_{i+1}(t)\right|\right\}$. Since $\Phi \in \Pi\left[L_{1}^{n}[a, b]\right]$, we see that the sequence $\tilde{\varphi}_{i} \in \Phi, i=1,2, \ldots$, has the following properties: for almost all $t \in[a, b]$, the inequalities (2.3) hold and $\left\|\tilde{\varphi}_{i}\right\|_{L_{1}^{n}[a, b]} \geqslant$ $\left\|\varphi_{i}\right\|_{L_{1}^{n}[a, b]}$ for each $i=1,2, \ldots$. Hence, from this property and equality (2.2), it follows that the sequence $\tilde{\varphi}_{i}, i=1,2, \ldots$, satisfies (2.2). Further, we consider a measurable function $u:[a, b] \rightarrow$ $[0, \infty)$ defined by

$$
u(t)=\lim _{i \rightarrow \infty}\left|\tilde{\varphi}_{i}(t)\right|
$$

Since the set $\Phi$ is bounded, we see, using Fatou's lemma (see [22]), that $u \in L_{1}^{1}[a, b]$. Moreover, by the definition of the function $u$ and due to (2.2),

$$
\int_{\mathcal{U}} u(t) d t=\|\Phi\|_{L_{1}^{n}(\mathcal{U})}
$$

for every measurable set $u \subset[a, b]$. Now, let us show that the function $u$ defined by (2.4) satisfies the assumptions of the lemma. Indeed, if the contrary is true, then there exist a function $\varphi \in \Phi$ and a measurable set $\mathcal{U}_{1} \subset[a, b]\left(\mu\left(\mathcal{U}_{1}\right)>0\right)$ such that $|\varphi(t)|>\boldsymbol{u}(t)$ for each $t \in \mathcal{U}_{1}$. This implies that $\int_{\mathcal{U}_{1}}\left|\varphi_{1}(t)\right| d t>\int_{\mathfrak{U}_{1}} u(t) d t$, which contradicts (2.5). This completes the proof.

Lemma 2.2. Let $\Phi \in \Pi\left[L_{1}^{n}[a, b]\right]$ and $\varphi_{i} \in \Phi, i=1,2, \ldots$, be a sequence that is dense in $\Phi$. Further, let a measurable set $F:[a, b] \rightarrow \operatorname{comp}\left[\mathbb{R}^{n}\right]$ be defined by

$$
F(t)=\overline{\left\{\varphi_{i}(t), i=1,2, \ldots\right\}}
$$

Then $S(F)=\Phi$.

Proof. Since $\varphi_{i} \in S(F)$ and the sequence $\varphi_{i}, i=1,2, \ldots$, is dense in $\Phi$, we have, due to the closedness of the set $\Phi$, the relation $\Phi \subset S(F)$. Let us prove that $S(F) \subset \Phi$. Let $x \in S(F)$. For each $k, i=1,2, \ldots$, put

$$
E_{i}^{k}=\left\{t \in[a, b]:\left|x(t)-\varphi_{i}(t)\right| \leqslant \frac{1}{k}\right\}
$$


which are measurable sets. For $i=1$, let $\widetilde{E}_{1}^{k}=E_{1}^{k}$, and for $i=2,3, \ldots$, let $\widetilde{E}_{i}^{k}=E_{i}^{k} \backslash \bigcup_{j=1}^{i-1} E_{j}^{k}$. Then $\widetilde{E}_{i}^{k} \cap \widetilde{E}_{j}^{k}=\varnothing$ if $i \neq j$. By the definition of the mapping $F:[a, b] \rightarrow \operatorname{comp}\left[\mathbb{R}^{n}\right]$, for each $k=1,2, \ldots$, we have

$$
\mu\left(\bigcup_{i=1}^{\infty} \widetilde{E}_{i}^{k}\right)=b-a
$$

Let $x_{k}:[a, b] \rightarrow \mathbb{R}^{n}, k=1,2, \ldots$, be a sequence of measurable functions such that

$$
x_{k}(t)= \begin{cases}\varphi_{i}(t) & \text { if } t \in \widetilde{E}_{i}^{k}, i=1,2, \ldots, k \\ \varphi_{1}(t) & \text { if } t \in[a, b] \backslash \bigcup_{i=1}^{k} \widetilde{E}_{i}^{k}\end{cases}
$$

Since the set $\Phi$ is decomposable, we see that $x_{k} \in \Phi$ for each $k=1,2, \ldots$ Moreover, from Lemma 2.1 and the definition of the set $\widetilde{E}_{i}^{k}$, it follows that for the functions $x_{k}, k=1,2, \ldots$, we have the estimates

$$
\left\|x-x_{k}\right\|_{L_{1}^{n}[a, b]} \leqslant \frac{b-a}{k}+2 \int_{[a, b] \backslash \cup_{i=1}^{k} \tilde{E}_{i}^{k}} u(t) d t
$$

where $u$ satisfies the assertions of Lemma 2.1. From (2.8) and (2.10), it follows that $x_{k} \rightarrow x$ in $L^{n}[a, b]$ as $k \rightarrow \infty$. Since the set $\Phi$ is closed, we have that $x \in \Phi$. Hence $S(F) \subset \Phi$. Thus $S(F)=\Phi$.

Lemma 2.3. Let measurable sets $F_{i}:[a, b] \rightarrow \operatorname{comp}\left[\mathbb{R}^{n}\right], i=1,2, \ldots$, be integrally bounded, then $S\left(F_{1}(\cdot)\right) \subset S\left(F_{2}(\cdot)\right)$ if and only if $F_{1}(t) \subset F_{2}(t)$ for almost all $t \in[a, b]$.

Proof. First of all, it is evident that if for almost all $t \in[a, b], F_{1}(t) \subset F_{2}(t)$, then $S\left(F_{1}(\cdot)\right) \subset$ $S\left(F_{2}(\cdot)\right)$.

Let $S\left(F_{1}(\cdot)\right) \subset S\left(F_{2}(\cdot)\right)$ and let $\varphi_{i} \in L_{1}^{n}[a, b], i=1,2, \ldots$, be a countable set, which is dense in $S\left(F_{1}\right)$ and which approximates $F_{1}:[a, b] \rightarrow \operatorname{comp}\left[\mathbb{R}^{n}\right]$ (see [21]). Thus $\varphi_{i} \in S\left(F_{2}(\cdot)\right)$ for each $i=1,2, \ldots$ and by the definition of the set $S\left(F_{2}(\cdot)\right)$, we have that $\left\{\varphi_{i}(t): i=1,2, \ldots\right\} \subset F_{2}(t)$ for almost all $t \in[a, b]$. Since the sequence $\varphi_{i}, i=1,2, \ldots$, approximates the map $F_{1}:[a, b] \rightarrow$ comp $\left[\mathbb{R}^{n}\right]$, it follows from the previous inclusion that $F_{1}(t) \subset F_{2}(t)$ for almost all $t \in[a, b]$.

Corollary 2.4. Let $\Phi \in \Pi\left[L_{1}^{n}[a, b]\right]$ and let $F_{i}:[a, b] \rightarrow \operatorname{comp}\left[\mathbb{R}^{n}\right], i=1,2$, be measurable sets such that $\Phi=S\left(F_{1}\right)=S\left(F_{2}\right)$. Then $F_{1}(t)=F_{2}(t)$ for almost all $t \in[a, b]$.

Remark 2.5. If $\Phi \in \Pi\left[L_{1}^{n}[a, b]\right]$, then a measurable set $F:[a, b] \rightarrow \operatorname{comp}\left[\mathbb{R}^{n}\right]$, that satisfies $S(F)=\Phi$, uniquely determines the set $\Phi$.

\section{Decomposable hull of a set in the space of integrable functions}

We introduce the concept of the decomposable hull of a set in the space $\mathbf{L}_{1}^{n}[a, b]$. We consider a multivalued mapping that is not necessarily decomposable. For such a mapping, we construct its decomposable hull and investigate topological properties of this hull. 
Definition 3.1. Let $\Phi$ be a nonempty subset of $\mathbf{L}_{1}^{n}[a, b]$. By dec $\Phi$, we denote the set of all finite combinations

$$
y=\chi\left(\mathfrak{U}_{1}\right) x_{1}+\chi\left(\mathfrak{U}_{2}\right) x_{2}+\cdots+\chi\left(\mathfrak{U}_{m}\right) x_{m}
$$

of elements $x_{i} \in \Phi, i=1,2, \ldots, m$, where the disjoint measurable subsets $\mathfrak{U}_{i}, i=1,2, \ldots, m$, of the segment $[a, b]$ are such that $\bigcup_{i=1}^{m} u_{i}=[a, b]$.

Lemma 3.2. The set dec $\Phi$ is decomposable for any nonempty set $\Phi \subset \mathbf{L}_{1}^{n}[a, b]$.

Proof. Let $y_{1}, y_{2} \in \operatorname{dec} \Phi$. Let also $u \subset[a, b]$ be a measurable set. Without loss of generality, it can be assumed that

$$
y_{i}=\chi\left(\mathcal{U}_{1}^{i}\right) x_{1}^{i}+\chi\left(\mathcal{U}_{2}^{i}\right) x_{2}^{i}+\cdots+\chi\left(\mathcal{U}_{m}^{i}\right) x_{m}^{i}
$$

where $x_{j}^{i} \in \Phi, j=1,2, \ldots, m, i=1,2$, and the measurable disjoint sets $\mathcal{U}_{j}^{i} \subset[a, b], j=1,2, \ldots, m$, $i=1,2$, are such that $[a, b]=\bigcup_{j=1}^{m} \mathfrak{U}_{j}^{i}, i=1,2$, (if the number of summands in (3.2) is not the same, we may use arbitrary functions multiplied by the characteristic functions of the empty sets). Further, from the equality

$$
\chi(\mathfrak{U}) y_{1}+\chi([a, b] \backslash \mathfrak{U}) y_{2}=\sum_{i=1}^{m} \chi\left(\mathcal{U} \cap \mathfrak{U}_{i}^{1}\right) x_{i}^{1}+\sum_{i=1}^{m} \chi\left(([a, b] \backslash \mathfrak{u}) \cap \mathfrak{U}_{i}^{2}\right) x_{i}^{2}
$$

it follows that $\chi(\mathcal{U}) y_{1}+\chi([a, b] \backslash \mathcal{U}) y_{2} \in \operatorname{dec} \Phi$. Hence, the set $\operatorname{dec} \Phi$ is decomposable.

Remark 3.3. Note that even if a set $\Phi \subset \mathbf{L}_{1}^{n}[a, b]$ is bounded, the set $\operatorname{dec} \Phi$ is not necessarily bounded. For example, let us check that

$$
\operatorname{dec}\left[B_{\mathbf{L}_{p}^{n}[a, b]}[0,1]\right]=\mathbf{L}_{p}^{n}[a, b] \quad(p \in[1, \infty)) .
$$

Indeed, let $z \in \mathbf{L}_{p}^{n}[a, b]$ and $e_{i}, i=1,2, \ldots, m$, be measurable sets with the following properties: $e_{i} \cap e_{j}=\varnothing$ if $i \neq j, i, j=1,2, \ldots, m, \bigcup_{i=1}^{m} e_{i}=[a, b]$; for each $i=1,2, \ldots, m$, the inequality

$$
\int_{e_{i}}|z(s)|^{p} d s<1
$$

holds. Then $z_{i}=\chi\left(e_{i}\right) z \in B_{\mathbf{L}_{p}^{n}[a, b]}[0,1], i=1,2, \ldots, m$, and

$$
z=x\left(e_{1}\right) z_{1}+x\left(e_{2}\right) z_{2}+\cdots+x\left(e_{m}\right) z_{m}
$$

Therefore, $z \in \operatorname{dec}\left[B_{\mathrm{L}_{p}^{n}[a, b]}[0,1]\right]$ and consequently, the equality (3.4) holds.

Remark 3.4. From (3.4), it follows that if a set $\Phi \subset \mathbf{L}_{1}^{n}[a, b]$ is relatively compact in the weak topology of $\mathbf{L}_{1}^{n}[a, b]$, then the set $\operatorname{dec} \Phi$ does not necessarily possess this property.

Remark 3.5. Note that if a set is convex in $\mathbf{L}_{1}^{n}[a, b]$, then this set is not necessarily decomposable. The ball $B_{\mathbf{L}_{1}^{n}[a, b]}[0,1]$ is an example of such a set. 
Remark 3.6. If a set $\Phi \subset \mathbf{L}_{1}^{n}[a, b]$ is integrally bounded, then by Lemma 2.2, for the set $\overline{\operatorname{dec}} \Phi \in \Pi\left[\mathbf{L}_{1}^{n}[a, b]\right]$, there exists a measurable and integrally bounded mapping $F_{\overline{\operatorname{dec}} \Phi}$ : $[a, b] \rightarrow \operatorname{comp}\left[R^{n}\right]$ such that

$$
\overline{\operatorname{dec}} \Phi=S\left(F_{\overline{\operatorname{dec}} \Phi}(\cdot)\right)
$$

Lemma 3.7. If a set $\Phi \subset \mathbf{L}_{1}^{n}[a, b]$ is decomposable, then $\operatorname{dec} \Phi=\Phi$.

Proof. Evidently, $\Phi \subset \operatorname{dec} \Phi$. We claim that $\operatorname{dec} \Phi \subset \Phi$. The proof is made by induction over $m$. By the definition of the switching convexity, any expression (3.1) including two elements $x_{1}, x_{2} \in \Phi$ and two measurable sets $\mathfrak{U}_{1}, \mathfrak{U}_{2} \subset[a, b]$ belongs to $\Phi$.

Suppose now that for $m=k$, the combination of the form (3.1) belongs to $\Phi$. Let $x_{1}, x_{2}, \ldots, x_{m+1} \in \Phi$ and let $\mathfrak{U}_{1}, \mathfrak{U}_{2}, \ldots, \mathfrak{U}_{m+1} \subset[a, b]$ be disjoint measurable sets such that $[a, b]=\bigcup_{i=1}^{m+1} \mathfrak{U}_{i}$. Let

$$
z=\chi\left(\mathfrak{U}_{2} \cup \mathfrak{U}_{1}\right) x_{2}+\chi\left(\mathfrak{U}_{3}\right) x_{3}+\cdots+\chi\left(\mathfrak{U}_{m+1}\right) x_{m+1}
$$

By the inductive assumption, $z \in \Phi$ and therefore $\chi\left(\mathfrak{U}_{1}\right) x_{1}+\chi\left([a, b] \backslash \mathfrak{U}_{1}\right) z \in \Phi$. Since

$$
x\left([a, b] \backslash \mathfrak{U}_{1}\right) z=x\left(\mathfrak{U}_{2}\right) x_{2}+\chi\left(\mathfrak{U}_{3}\right) x_{3}+\cdots+\chi\left(\mathfrak{U}_{m+1}\right) x_{m+1},
$$

we have that

$$
\chi\left(\mathfrak{U}_{1}\right) x_{1}+\chi\left(\mathfrak{U}_{2}\right) x_{2}+\cdots+\chi\left(\mathfrak{U}_{m+1}\right) x_{m+1} \in \Phi
$$

Hence dec $\Phi \subset \Phi$. This concludes the proof.

Corollary 3.8. If $\Phi \subset \mathbf{L}_{1}^{n}[a, b]$, then the set $\operatorname{dec} \Phi$ is the minimal set which is decomposable and which contains $\Phi$.

Proof. Consider any set $U \subset \mathbf{L}_{1}^{n}[a, b]$ which is decomposable and which satisfies $\Phi \subset U$. Then, by Lemma 3.7, we have $\Phi \subset \operatorname{dec} \Phi \subset \operatorname{dec} U=U$.

Lemma 3.9. If a set $\Phi \subset \mathbf{L}_{1}^{n}[a, b]$ is convex, then so is the set $\operatorname{dec} \Phi \in \mathbf{L}_{1}^{n}[a, b]$.

Proof. Let $y_{1}, y_{2} \in \operatorname{dec} \Phi$ be given by the formula (3.2). It follows from the convexity of the set $\Phi \subset \mathbf{L}_{1}^{n}[a, b]$ and the equality

$$
\lambda y_{1}+(1-\lambda) y_{2}=\sum_{i, j=1}^{m} x\left(\mathcal{U}_{i}^{1} \cap \mathcal{U}_{j}^{2}\right)\left(\lambda x_{i}^{1}+(1-\lambda) x_{j}^{2}\right)
$$

that $\lambda y_{1}+(1-\lambda) y_{2} \in \operatorname{dec} \Phi$ for any $\lambda \in[0,1]$. Thus, the set $\operatorname{dec} \Phi$ is convex.

Similar to the definition of the convex hull in a normed space, the set $\operatorname{dec} \Phi$ will, in the sequel, be called the decomposable hull of the set $\Phi$ in the space of integrable functions, or simply the decomposable hull of the set $\Phi$. Likewise, $\overline{\operatorname{dec}} \Phi$ is addressed as the closed decomposable hull of the set $\Phi$. 
Remark 3.10. If $\Phi \in Q\left[\mathbf{L}_{1}^{n}[a, b]\right]$, then the closed decomposable hull of the set $\Phi$ (the set $\overline{\operatorname{dec}} \Phi$ ) can be constructed as described in Remark 3.6. To do it, one needs a measurable and integrally bounded (see Remark 3.6) mapping $F_{\overline{\operatorname{dec}} \Phi}:[a, b] \rightarrow \operatorname{comp}\left[\mathbb{R}^{n}\right]$ that satisfies (3.7). Note that finding this mapping $F_{\overline{\operatorname{dec}} \Phi}$ is easier than constructing the set $\overline{\operatorname{dec}} \Phi$. At the same time, when one studies the metrical relations between the sets $\Phi_{1}, \Phi_{2} \subset \mathbf{L}_{1}^{n}[a, b]$ and their decomposable hulls (see Lemma 3.12), it is more convenient to use Definition 3.1.

Lemma 3.11. Let $v \in \mathbf{L}_{1}^{n}(\mathcal{U})(\mathcal{U} \subset[a, b])$ and let a set $\Phi \subset \mathbf{L}_{1}^{n}[a, b]$ be decomposable. Then for any disjoint measurable sets $\mathfrak{U}_{1}, \mathfrak{U}_{2} \subset \boldsymbol{U}$ such that $\mathfrak{U}_{1} \cup \mathfrak{U}_{2}=\mathfrak{U}_{\text {, one has }}$

$$
\rho_{\mathbf{L}_{1}^{n}(\mathcal{U})}[v ; \Phi]=\rho_{\mathbf{L}_{1}^{n}\left(\mathcal{U}_{1}\right)}[v ; \Phi]+\rho_{\mathbf{L}_{1}^{n}\left(\mathcal{U}_{2}\right)}[v ; \Phi] .
$$

Proof. Indeed, let $\varepsilon>0$ and $y \in \Phi$ satisfy $\|v-y\|_{\mathbf{L}_{1}^{n}(\mathcal{U})}<\rho_{\mathbf{L}_{1}^{n}(\mathcal{U})}[v ; \Phi]+\varepsilon$. It follows from this estimate that

$$
\rho_{\mathbf{L}_{1}^{n}\left(\mathcal{U}_{1}\right)}[v ; \Phi]+\rho_{\mathbf{L}_{1}^{n}\left(\mathcal{U}_{2}\right)}[v ; \Phi] \leqslant\|v-y\|_{\mathbf{L}_{1}^{n}\left(\mathcal{U}_{1}\right)}+\|v-y\|_{\mathbf{L}_{1}^{n}\left(\mathcal{U}_{2}\right)}<\rho_{\mathbf{L}_{1}^{n}(\mathcal{U})}[v ; \Phi]+\varepsilon .
$$

This yields

$$
\rho_{\mathbf{L}_{1}^{n}\left(\mathcal{U}_{1}\right)}[v ; \Phi]+\rho_{\mathbf{L}_{1}^{n}\left(\mathcal{U}_{2}\right)}[v ; \Phi] \leqslant \rho_{\mathbf{L}_{1}^{n}(\mathcal{U})}[v ; \Phi] .
$$

Further, let us show that the opposite inequality is valid. Let $y_{i} \in \Phi{\mid \mathfrak{U}_{i}}, i=1,2$, where $\left.\Phi\right|_{\mathcal{U}_{i}}$ is the set of of all mappings from $\Phi$, restricted to $\mathcal{U}_{i}, i=1,2$, and suppose that the functions $y_{i}, i=1,2$, satisfy

$$
\left\|v-y_{i}\right\|_{\mathbf{L}_{1}^{n}\left(\mathcal{U}_{i}\right)}<\rho_{\mathbf{L}_{1}^{n}\left(\mathcal{U}_{i}\right)}[v ; \Phi]+\frac{\varepsilon}{2}, \quad i=1,2 .
$$

Since the set $\Phi$ is decomposable, it follows that the map $y: \mathfrak{U} \rightarrow \mathbb{R}$ defined by

$$
y(t)= \begin{cases}y_{1}(t) & \text { if } t \in \mathcal{U}_{1} \\ y_{2}(t) & \text { if } t \in \mathcal{U}_{2}\end{cases}
$$

belongs to the set $\left.\Phi\right|_{\mathfrak{u}}$. By (3.15), we have

$$
\rho_{\mathbf{L}_{1}^{n}(\mathcal{U})}[v ; \Phi] \leqslant\|v-y\|_{\mathbf{L}_{1}^{n}(\mathcal{U})}<\rho_{\mathbf{L}_{1}^{n}\left(\mathcal{U}_{1}\right)}[v ; \Phi]+\rho_{\mathbf{L}_{1}^{n}\left(\mathcal{U}_{2}\right)}[v ; \Phi]+\varepsilon .
$$

This implies that

$$
\rho_{\mathbf{L}_{1}^{n}(\mathcal{U})}[v ; \Phi] \leqslant \rho_{\mathbf{L}_{1}^{n}\left(\mathcal{U}_{1}\right)}[v ; \Phi]+\rho_{\mathbf{L}_{1}^{n}\left(\mathcal{U}_{2}\right)}[v ; \Phi] .
$$

Comparing (3.14) and (3.18), we obtain (3.12).

Lemma 3.12. If $\Phi_{1}, \Phi_{2} \in Q\left[\mathbf{L}_{1}^{n}[a, b]\right]$ and there exists a function $\omega \in \mathbf{L}_{+}^{1}[a, b]$ such that

$$
h_{\mathrm{L}_{1}^{n}(\mathcal{U})}^{+}\left[\Phi_{1} ; \Phi_{2}\right] \leqslant \int_{\mathcal{U}} \omega(s) d s
$$

for any measurable set $\mathfrak{U} \subset[a, b]$, then

$$
h_{\mathrm{L}_{1}^{n}(\mathcal{U})}^{+}\left[\operatorname{dec} \Phi_{1} ; \operatorname{dec} \Phi_{2}\right] \leqslant \int_{\mathcal{U}} \omega(s) d s
$$

for any measurable set $\mathcal{U} \subset[a, b]$. 
Proof. Let $\mathcal{U} \subset[a, b]$ be a measurable set, $\mu(\mathcal{U})>0$. Let $z \in \operatorname{dec} \Phi_{1}$ and $z_{i} \in \Phi_{1}, i=1,2, \ldots, m$. Suppose also that the functions $z_{i}$ and disjoint measurable sets $\tilde{e}_{i} \subset[a, b], i=1,2, \ldots, m$, such that $[a, b]=\bigcup_{i=1}^{m} \tilde{e}_{i}$, satisfy the equality

$$
z=x\left(\tilde{e}_{1}\right) z_{1}+\chi\left(\tilde{e}_{2}\right) z_{2}+\cdots+\chi\left(\tilde{e}_{m}\right) z_{m} .
$$

Further, by $z, z_{i}, i=1,2, \ldots, m$, we denote the restrictions of these functions to $\mathcal{U}$ and put $e_{i}=\tilde{e}_{i} \cap \mathcal{U}, i=1,2, \ldots, m$.

From (3.21) and Lemma 3.11, it follows that

$$
\rho_{\mathbf{L}_{1}^{n}(\mathcal{U})}\left[z ; \operatorname{dec} \Phi_{2}\right]=\sum_{i=1}^{m} \rho_{\mathbf{L}_{1}^{n}\left(e_{i}\right)}\left[z_{i} ; \operatorname{dec} \Phi_{2}\right] \leqslant \sum_{i=1}^{m} \rho_{\mathbf{L}_{1}^{n}\left(e_{i}\right)}\left[z_{i} ; \Phi_{2}\right] .
$$

From (3.19), we obtain that

$$
\rho_{\mathbf{L}_{1}^{n}\left(e_{i}\right)}\left[z_{i} ; \Phi_{2}\right] \leqslant \int_{e_{i}} \omega(s) d s
$$

for each $i=1,2, \ldots, m$.

Therefore, (3.22) and (3.23) imply

$$
\rho_{\mathbf{L}_{1}^{n}(\mathcal{U})}\left[z ; \operatorname{dec} \Phi_{2}\right] \leqslant \int_{\mathcal{U}} \omega(s) d s .
$$

Since (3.24) holds for any $z \in \operatorname{dec} \Phi_{1}$, it follows from (3.24) that (3.20) holds as well.

Remark 3.13. Note that the function $\omega \in \mathbf{L}_{+}^{1}[a, b]$ (see (3.19)) provides a uniform with respect to measurable sets $U \subset[a, b]$ estimate for the Hausdorff semideviation of the set $\Phi_{1}$ from the set $\Phi_{2}$.

Remark 3.14. The inequality (3.20) holds true even if the set $\operatorname{dec} \Phi_{i}$ is replaced with its closure $\overline{\operatorname{dec}} \Phi_{i}, i=1,2$.

We say that a multivalued mapping $\Phi: \mathbf{C}^{n}[a, b] \rightarrow Q\left[\mathbf{L}_{1}^{n}[a, b]\right]$ is integrally bounded on $a$ set $K \subset \mathbf{C}^{n}[a, b]$ if the image $\Phi(K)$ is integrally bounded.

Let $\Phi: \mathbf{C}^{n}[a, b] \rightarrow Q\left[\mathbf{L}_{1}^{n}[a, b]\right]$. We introduce an operator $\widetilde{\Phi}: \mathbf{C}^{n}[a, b] \rightarrow \Pi\left[\mathbf{L}_{1}^{n}[a, b]\right]$ by the formula

$$
\widetilde{\Phi}(x)=\overline{\operatorname{dec}} \Phi(x) .
$$

Note that even if a mapping $\Phi: \mathbf{C}^{n}[a, b] \rightarrow Q\left[\mathbf{L}_{1}^{n}[a, b]\right]$ is continuous, the mapping $\widetilde{\Phi}: \mathbf{C}^{n}[a, b] \rightarrow \Pi\left[\mathbf{L}_{1}^{n}[a, b]\right]$ given by (3.25) may be discontinuous. To illustrate this, let us consider an example.

Example 3.15. We define an integrable function $\varphi:[0,2] \times[0,1] \times[0,2] \rightarrow \mathbb{R}^{1}$ by

$$
\varphi(x, r)(t)= \begin{cases}1 & \text { if } t \in[x, x+r] \cap[0,2], r \neq 0, \\ 0 & \text { if } t \bar{\in}[x, x+r] \cap[0,2], r \neq 0, \\ 0 & \text { if } r=0 .\end{cases}
$$




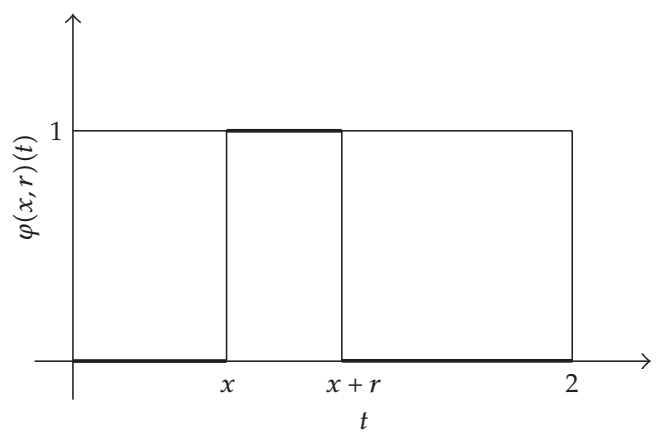

Figure 1

We also define a multivalued mapping $\Phi:[0,1] \rightarrow Q\left[L_{1}^{1}[0,2]\right]$ by the formula

$$
\Phi(r)=\left\{\begin{array}{cc}
\bigcup_{x \in[0,2]} \varphi(x, r) & \text { if } r \neq 0, \\
0 & \text { if } r=0 .
\end{array}\right.
$$

Note that

$$
h_{L_{1}^{1}[0,2]}\left[\Phi\left(r_{1}\right) ; \Phi\left(r_{2}\right)\right]=\left|r_{1}-r_{2}\right|
$$

for any $r_{1}, r_{2} \in[0,1]$, but at the same time,

$$
h_{L_{1}^{1}[0,2]}[\tilde{\Phi}(0) ; \tilde{\Phi}(r)]=2
$$

for any $r \in(0,1]$.

Using Lemma 3.12, we obtain the following continuity conditions for the operator $\widetilde{\Phi}$ : $\mathbf{C}^{n}[a, b] \rightarrow \Pi\left[\mathbf{L}_{1}^{n}[a, b]\right]$ given by (3.25).

Definition 3.16. Let $U \subset C^{n}[a, b]$. One says that a mapping $P: U \times U \rightarrow L_{+}^{1}[a, b]$ is symmetric on the set $U$ if $P(x, y)=P(y, x)$ for any $x, y \in U$. One says that a mapping $P: U \times U \rightarrow L_{+}^{1}[a, b]$ is continuous in the second variable at a point $(x, x)$ belonging to the diagonal of $U \times U$ if for any sequence $y_{i} \in U$ such that $y_{i} \rightarrow x$ as $i \rightarrow \infty$ it holds that $P(x, x)=\lim _{i \rightarrow \infty} P\left(x, y_{i}\right)$. One says that a mapping $P: U \times U \rightarrow L_{+}^{1}[a, b]$ is continuous in the second variable on the diagonal of $U \times U$ if $P$ is continuous in the second variable at each point of this diagonal. Continuity in the fist variable is defined similarly.

Definition 3.17. Let $U \subset C^{n}[a, b]$. Suppose also that $P(x, x)=0$ for any $x \in U$. One says that a mapping $P: U \times U \rightarrow L_{+}^{1}[a, b]$ has property $\mathcal{A}$ on the set $U$ if it is continuous in the second variable on the diagonal of $U \times U$; it has property $B$ on the set $U$ if it is continuous in the first variable on the diagonal of $U \times U$; it has property $\mathcal{C}$ on the set $U$ if it is continuous on the diagonal of $U \times U$ and symmetric on the set $U$.

Theorem 3.18. Let $U \subset C^{n}[a, b]$. Suppose also that for a mapping $\Phi: \mathbf{C}^{n}[a, b] \rightarrow Q\left[\mathbf{L}_{1}^{n}[a, b]\right]$ there exists a mapping $P: U \times U \rightarrow L_{+}^{1}[a, b]$ such that

$$
h_{L_{1}^{n}(\mathcal{U})}^{+}[\Phi(x), \Phi(y)] \leqslant\|P(x, y)\|_{L_{1}^{1}(\mathcal{U})}
$$


for any $x, y \in U$ and any measurable set $\boldsymbol{U} \subset[a, b]$. Then for the mapping $\widetilde{\Phi}: \mathbf{C}^{n}[a, b] \rightarrow \Pi\left[\mathbf{L}_{1}^{n}[a, b]\right]$ given by (3.25), the inequality (3.30), where $\Phi(\cdot) \equiv \widetilde{\Phi}(\cdot)$, is satisfied as well as for any $x, y \in U$ and any measurable set $\boldsymbol{U} \subset[a, b]$.

Corollary 3.19. If the mapping $P: U \times U \rightarrow L_{+}^{1}[a, b]$ in Theorem 3.18 has property $\mathbb{A}$ (resp., $\mathbb{B}, \mathcal{C}$ ) on the set $U \subset C^{n}[a, b]$, then the operator $\widetilde{\Phi}: C^{n}[a, b] \rightarrow \Pi\left[L_{1}^{n}[a, b]\right]$ given by (3.25) is Hausdorff lower semicontinuous (resp., Hausdorff upper semicontinuous, Hausdorff continuous) on the set $U \subset$ $C^{n}[a, b]$.

We say that the mapping $P: U \times U \rightarrow L_{+}^{1}[a, b]$ satisfying the inequality (3.30) for any measurable set $\mathcal{U} \subset[a, b]$ is a majorant mapping for $\Phi: C^{n}[a, b] \rightarrow Q\left[L_{1}^{n}[a, b]\right]$ on the set $U$.

Let a mapping $F_{i}:[a, b] \times \mathbb{R}^{n} \rightarrow \operatorname{comp}\left[\mathbb{R}^{n}\right], i=1,2$, be measurable as a composite function for every $x \in C^{n}[a, b]$. Let also $F_{i}$ be integrally bounded for every bounded set $K \subset \mathbb{R}^{n}$. Consider a mapping $\mathcal{M}: C^{n}[a, b] \rightarrow Q\left[L_{1}^{n}[a, b]\right]$ given by

$$
\mathcal{M}(x)=\mathcal{N}_{1}(x) \cup \mathcal{N}_{2}(x),
$$

where the mapping $\mathcal{N}_{i}: C^{n}[a, b] \rightarrow \Pi\left[L_{1}^{n}[a, b]\right], i=1,2$, is the Nemytskii operator generated by the mapping $F_{i}:[a, b] \times \mathbb{R}^{n} \rightarrow \operatorname{comp}\left[\mathbb{R}^{n}\right], i=1,2$. For the operator $\mathcal{M}: C^{n}[a, b] \rightarrow$ $Q\left[L_{1}^{n}[a, b]\right]$ given by (3.31), the majorant mapping $\widetilde{P}: C^{n}[a, b] \times C^{n}[a, b] \rightarrow L_{+}^{1}[a, b]$ can be defined as

$$
\widetilde{P}(x, y)(t)=\max \left\{h^{+}\left[F_{1}(t, x(t)) ; F_{1}(t, y(t))\right] ; h^{+}\left[F_{2}(t, x(t)) ; F_{2}(t, y(t))\right]\right\} .
$$

It follows from Theorem 3.18 that the operator $\widetilde{P}(\cdot, \cdot)$ given by $(3.32)$ is also a majorant mapping for the mapping $\widetilde{\mathcal{M}}: C^{n}[a, b] \rightarrow \Pi\left[L_{1}^{n}[a, b]\right]$ given by (3.25), where $\Phi(\cdot) \equiv \mathcal{M}(\cdot)$. If the mapping $F_{i}:[a, b] \times \mathbb{R}^{n} \rightarrow \operatorname{comp}\left[\mathbb{R}^{n}\right], i=1,2$, is Hausdorff lower semicontinuous (resp., Hausdorff upper semicontinuous and Hausdorff continuous) in the second variable, then by Corollary 3.19, the mapping $\widetilde{M}: C^{n}[a, b] \rightarrow \Pi\left[L_{1}^{n}[a, b]\right]$ given by (3.25) is Hausdorff lower semicontinuous (resp., Hausdorff upper semicontinuous and Hausdorff continuous).

Definition 3.20. One says that a multivalued mapping $\Phi: C^{n}[a, b] \rightarrow Q\left[L_{1}^{n}[a, b]\right]$ has Property $\mathcal{A}\left(\right.$ resp., $B$ and $\mathcal{C}$ ) if for this mapping there exists a majorant mapping $P: C^{n}[a, b] \times C^{n}[a, b] \rightarrow$ $L_{+}^{1}[a, b]$ satisfying Property $\mathcal{A}$ (resp., $B$ and $\mathcal{C}$ ).

\section{Basic properties of generalized solutions of functional differential inclusions}

Using decomposable hulls, we introduce in this section the concept of a generalized solution of a functional differential inclusion with a right-hand side which is not necessarily decomposable. Using, as mentioned in Section 3, basic topological properties of a mapping given by (3.25), we study the properties of a generalized solution of the initial value problem.

Consider the initial value problem for the functional differential inclusion

$$
\dot{x} \in \Phi(x), \quad x(a)=x_{0} \quad\left(x_{0} \in \mathbb{R}^{n}\right),
$$

where the mapping $\Phi: \mathbf{C}^{n}[a, b] \rightarrow Q\left[\mathbf{L}_{1}^{n}[a, b]\right]$ satisfies the following condition: for every bounded set $U \subset \mathbf{C}^{n}[a, b]$, the image $\Phi(U)$ is integrally bounded. Note that the right-hand side 
of the inclusion (4.1) is not necessarily decomposable. Note also that $\dot{x}$ in (4.1) is not treated as a derivative at a point but as an element of $\mathbf{L}_{1}^{n}[a, b]$ (see [10, 23-25]). When we study such a problem, there may appear some difficulties described in the introduction. In this connection, we will introduce the concept of a generalized solution of the problem (4.1) and study the properties of this solution. Using the Nemytskii operator, which is decomposable, the initial value problem for a classical differential inclusion, that is, one without delay (see [10,23-25]), can be reduced to (4.1).

Definition 4.1. An absolutely continuous function $x:[a, b] \rightarrow \mathbb{R}^{n}$ is called a generalized solution of the problem (4.1) if

$$
\dot{x} \in \overline{\operatorname{dec}} \Phi(x), \quad x(a)=x_{0} \quad\left(x_{0} \in \mathbb{R}^{n}\right) .
$$

Note that from Lemma 3.7, it follows that if the set $\Phi(x)$ (see(4.1)) is decomposable, then a generalized solution of the problem (4.1) coincides with a classical solution.

Example 4.2. Consider an ordinary differential equation, $x \in[0,1]$,

$$
\dot{x}=k x, \quad x(0)=1 .
$$

Its solution is the function $x=e^{k t}$.

We assume that the parameter $k$ may take two values: 1 or 2 . Then the trajectories of such a system are described by the differential inclusion

$$
\dot{x} \in \Phi(t) x(t), \quad x(0)=1,
$$

where $\Phi(t)$ is a multivalued function with the values from the set $\{1,2\}$. Note that $\overline{\operatorname{dec}} \Phi(t)=\Phi(t)$, that is, the set in the right-hand side of the inclusion is decomposable. In this case, a generalized solution of the inclusion coincides with a classical solution.

The latter differential inclusion describes the model that is controlled by the differential equation either with the parameter value $k=1$ or with the parameter value $k=2$. In this model, switchings from one law (equation) to another may take place any time.

In the limit case, all possible solutions fill entirely the set of all points between the graphs of the functions $e^{t}$ and $e^{2 t}$.

Example 4.3. Consider a simple pendulum. It consists of a mass $m$ hanging from a string of length $l$ and fixed at a pivot point $P$. When displaced to an initial angle and released, the pendulum will swing back and forth with periodic motion. The equation of motion for the pendulum is given by

$$
\ddot{x}=-a \sin x,
$$

where $x(t)$ is the angular displacement at the moment $t, a=g / l, g$ is the acceleration of gravity, and $l$ is the length of the string.

If the amplitude of angular displacement is small enough that the small angle approximation holds true, then the equation of motion reduces to the equation of simple harmonic motion

$$
\ddot{x}=-a x .
$$




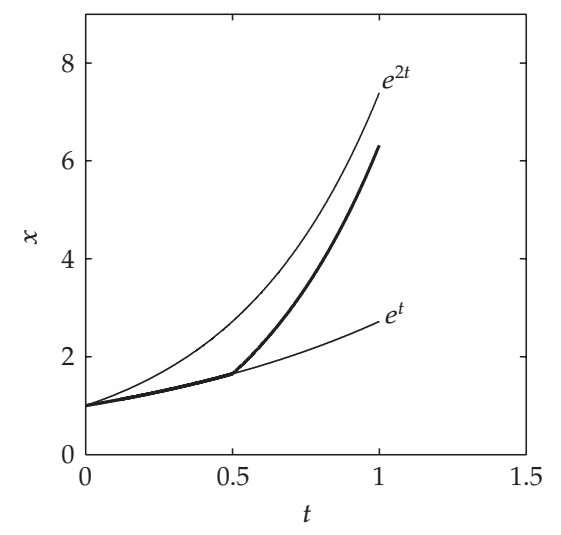

Figure 2: The solution of the differential inclusion that corresponds to switching from $k=1$ (control law 1 ) to $k=2$ (control law 2 ) at the moment $t=1 / 2$.

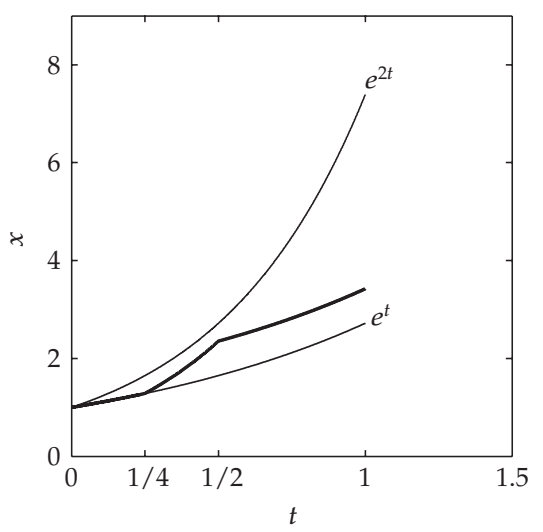

Figure 3: The solution of the differential inclusion that corresponds to two switchings: from $k=1$ to $k=2$ at the moment $t=1 / 4$ and from $k=2$ to $k=1$ at the moment $t=1 / 2$.

Let us now assume that the length of the string $l$ may change, that is, it may take an value from a finite set $\left\{l_{1}, \ldots, l_{m}\right\}$. In this case, the equation of simple harmonic motion transforms to the differential inclusion with a multivalued mapping

$$
\ddot{x} \in \Phi(x),
$$

where $\Phi(x)=\bigcup_{i=1}^{m}-\left(g / l_{i}\right) x$.

We assume that switching from one length (equation) to another may take place any time. Then the generalized solutions of the inclusion treat all available trajectories (states) corresponding to all switchings.

Definition 4.4. An operator $\Phi: \mathbf{C}^{n}[a, b] \rightarrow Q\left[\mathbf{L}_{1}^{n}[a, b]\right]$ is called a Volterra-Tikhonov (or simply a Volterra) operator (see [26]) if the equality $x=y$ on $[a, \tau], \tau \in(a, b]$, implies $\left.(\Phi(x))\right|_{\tau}=$ $\left.(\Phi(y))\right|_{\tau}$, where $\left.(\Phi(z))\right|_{\tau}$ is the set of all functions from $\Phi(z)$ restricted to $[a, \tau]$. 


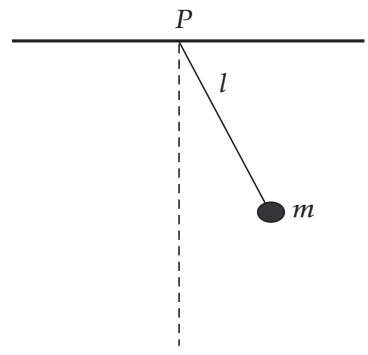

Figure 4

In what follows, we assume that the operator $\Phi: \mathbf{C}^{n}[a, b] \rightarrow Q\left[\mathbf{L}_{1}^{n}[a, b]\right]$ (the right-hand side of the inclusion (4.1)) is a Volterra operator. This implies that the operator $\widetilde{\Phi}: \mathbf{C}^{n}[a, b] \rightarrow$ $Q\left[\mathbf{L}_{1}^{n}[a, b]\right]$ given by (3.25) is also a Volterra operator.

Let $\tau \in(a, b]$. Let us determine the continuous mapping $V_{\tau}: \mathrm{C}^{n}[a, \tau] \rightarrow \mathrm{C}^{n}[a, b]$ by

$$
\left(V_{\tau} x\right)= \begin{cases}x(t) & \text { if } t \in[a, \tau] \\ x(\tau) & \text { if } t \in(\tau, b]\end{cases}
$$

Definition 4.5. One says that an absolutely continuous function $x:[a, \tau] \rightarrow \mathbb{R}^{n}$ is a generalized solution of the problem (4.1) on the interval $[a, \tau], \tau \in(a, b]$, if $x$ satisfies $\left.\dot{x} \in\left(\overline{\operatorname{dec}} \Phi\left(V_{\tau}(x)\right)\right)\right|_{\tau}$ and $x(a)=x_{0}$, where the continuous mapping $V_{\tau}: \mathbf{C}^{n}[a, \tau] \rightarrow \mathbf{C}^{n}[a, b]$ is given by (4.8).

A function $x:[a, c) \rightarrow \mathbb{R}^{n}$, which is absolutely continuous on any interval $[a, \tau] \subset[a, c)$, $c \in(a, b]$, is called a generalized solution of the problem (4.1) on the interval $[a, c)$ if for each $\tau \in(a, c)$ the restriction of $x$ to $[a, \tau]$ is a generalized solution of the problem (4.1) on the interval $[a, \tau]$.

A generalized solution $x:[a, c) \rightarrow \mathbb{R}^{n}$ of the problem (4.1) on the interval $[a, c)$ is said to be nonextendable if there is no generalized solution $y$ of the problem (4.1) on any larger interval $[a, \tau]$ (here, $\tau \in(c, b]$ if $c<b$ and $\tau=b$ if $c=b$ ) such that $x(t)=y(t)$ for each $t \in[a, c)$.

In Theorems 4.6-4.12 below, we assume that the mapping $\Phi: \mathbf{C}^{n}[a, b] \rightarrow Q\left[\mathbf{L}_{1}^{n}[a, b]\right]$ has Property $\mathcal{A}$. Due to Corollary 3.19, the mapping $\widetilde{\Phi}: \mathbf{C}^{n}[a, b] \rightarrow \Pi\left[\mathbf{L}_{1}^{n}[a, b]\right]$ given by (3.25) is lower semicontinuous. Due to $[27,28]$, the mapping $\widetilde{\Phi}: \mathbf{C}^{n}[a, b] \rightarrow \Pi\left[\mathbf{L}_{1}^{n}[a, b]\right]$ admits a continuous selection. Therefore, the following propositions on local solutions of the problem (4.1) are straightforward.

Theorem 4.6. There exists $\tau \in(a, b]$ such that a generalized solution of the problem (4.1) is defined on the interval $[a, \tau]$.

Theorem 4.7. A generalized solution $x:[a, c) \rightarrow \mathbb{R}^{n}$ of the problem (4.1) admits a continuation if and only if $\varlimsup_{t \rightarrow c-0}|x(t)|<\infty$.

Theorem 4.8. If $y$ is a generalized solution of the problem (4.1) on the interval $[a, \tau], \tau \in(a, b)$, then there exists a nonextendable solution $x$ of the problem (4.1) defined on the interval $[a, c)(c \in(\tau, b])$, or on the entire interval $[a, b]$, such that $x(t)=y(t)$ for each $t \in[a, \tau]$.

Let $H\left(x_{0}, \tau\right)$ be the set of all generalized solutions of the problem (4.1) on the interval $[a, \tau](\tau \in(a, b])$. 
We say that generalized solutions of the problem (4.1) admit a uniform a priori estimate if there exists a number $r>0$ such that for every $\tau \in(a, b]$, there is no generalized solution $y \in H\left(x_{0}, \tau\right)$ satisfying $\|y\|_{\mathbb{C}^{n}[a, \tau]}>r$.

Theorems 4.6-4.8 yield the following result.

Theorem 4.9. Let the generalized solutions of the problem (4.1) admit a uniform a priori estimate. Then $H\left(x_{0}, \tau\right) \neq \varnothing$ for any $\tau \in(a, b]$ and there exists a number $r>0$ such that $\|y\|_{\mathrm{C}^{n}[a, \tau]} \leqslant r$ for any $\tau \in(a, b], y \in H\left(x_{0}, \tau\right)$.

Definition 4.10. One says that a mapping $\Phi: \mathbf{C}^{n}[a, b] \rightarrow Q\left[\mathbf{L}_{1}^{n}[a, b]\right]$ has Property $\Gamma_{1}$ if there exists an isotonic continuous operator $\Gamma_{1}: \mathbf{C}_{+}^{1}[a, b] \rightarrow \mathbf{L}_{+}^{1}[a, b]$ satisfying the following conditions:

(i) for any function $x \in \mathrm{C}^{n}[a, b]$ and any measurable set $\mathcal{U} \subset[a, b]$, one has

$$
\|\Phi(x)\|_{L_{1}^{n}(\mathcal{U})} \leqslant\left\|\Gamma_{1}(Z x)\right\|_{\mathbf{L}_{1}^{1}(\mathcal{U})^{\prime}}
$$

where the continuous mapping $Z: \mathbf{C}^{n}[a, b] \rightarrow \mathbf{C}_{+}^{1}[a, b]$ is given by

$$
(Z x)(t)=|x(t)| ;
$$

(ii) the local solutions of the problem

$$
\dot{y}=\Gamma_{1}(y), \quad y(a)=\left|x_{0}\right|
$$

admit a uniform a priori estimate.

Lemma 4.11. Suppose that a multivalued mapping $\Phi: \mathbf{C}^{n}[a, b] \rightarrow Q\left[\mathbf{L}_{1}^{n}[a, b]\right]$ has Property $\Gamma_{1}$. Then so does the mapping $\widetilde{\Phi}: \mathbf{C}^{n}[a, b] \rightarrow \Pi\left[\mathbf{L}_{1}^{n}[a, b]\right]$ given by (3.25).

Proof. It suffices to show that

$$
\|\operatorname{dec} \Phi(x)\|_{\mathbf{L}_{1}^{n}(\mathcal{U})} \leqslant\left\|\Gamma_{1}(Z x)\right\|_{\mathbf{L}_{1}^{1}(\mathcal{U})}
$$

for any function $x \in \mathrm{C}^{n}[a, b]$ and any measurable set $\mathcal{U} \subset[a, b]$. Indeed, let a function $y \in$ $\operatorname{dec} \Phi(x)$ be as in (3.1). By (4.9),

$$
\int_{\mathcal{U}_{i} \cap \mathcal{U}}\left|x_{i}(s)\right| d s \leqslant\left\|\Gamma_{1}(Z x)\right\|_{\mathbf{L}_{1}^{1}\left(\mathcal{U}_{i} \cap \mathcal{U}\right)}
$$

for each $i=1,2, \ldots, m$. Hence, we have that for the function $y \in \operatorname{dec} \Phi(x)$, the estimate

$$
\int_{\mathcal{U}}|y(s)| d s \leqslant\left\|\Gamma_{1}(Z x)\right\|_{\mathbf{L}_{1}^{1}(\mathcal{U})}
$$

is satisfied as well. This gives the inequality (4.12). The proof is complete.

Let a continuous operator $\Theta: \mathbf{D}^{n}[a, b] \rightarrow \mathbf{C}_{+}^{1}[a, b]$ be given by

$$
(\Theta z)(t)=|z(a)|+\int_{a}^{t}|\dot{z}(s)| d s .
$$


Theorem 4.12. Suppose that a mapping $\Phi: \mathbf{C}^{n}[a, b] \rightarrow Q\left[\mathbf{L}_{1}^{n}[a, b]\right]$ has Property $\Gamma_{1}$. Then the set $H\left(x_{0}, \tau\right)$ is nonempty for any $\tau \in(a, b]$ and there exists a number $r>0$ such that $\|y\|_{\mathrm{C}^{n}[a, \tau]} \leqslant r$ for any $y \in H\left(x_{0}, \tau\right), \tau \in(a, b]$.

Proof. Indeed, let $x \in H\left(x_{0}, \tau\right)(\tau \in(a, b])$. From Lemma 4.11, it follows that for any $t \in[a, \tau]$,

$$
(\Theta x)(t) \leqslant\left|x_{0}\right|+\int_{a}^{t}\left(\Gamma_{1}(Z x)\right)(s) d s \leqslant\left|x_{0}\right|+\int_{a}^{t}\left(\Gamma_{1}(\Theta x)\right)(s) d s,
$$

where the function $\Theta x$ is given by (4.15). Due to the theorem on integral inequalities for an isotonic operator (see [29]), this implies that we actually have $\Theta x \leqslant \xi_{0}$, where $\xi_{0}$ is the upper solution of the problem (4.11). Thus, there is no $x \in H\left(x_{0}, \tau\right)$ satisfying the inequality $\|x\|_{C^{n}[a, \tau]}>\left\|\xi_{0}\right\|_{C^{1}[a, b]}$. From this, it follows that the set of all local generalized solutions of the problem (4.1) admits a uniform a priori estimate. Applying Theorem 4.9 completes the proof.

Let a linear continuous operator $\Lambda: \mathbf{L}_{1}^{n}[a, b] \rightarrow \mathbf{C}^{n}[a, b]$ be given by

$$
(\Lambda z)(t)=\int_{a}^{t} z(s) d s, \quad t \in[a, b]
$$

We say that $\Lambda: \mathbf{L}_{1}^{n}[a, b] \rightarrow \mathbf{C}^{n}[a, b]$ is the operator of integration.

Theorem 4.13. Let the set of all local generalized solutions of the problem (4.1) admit a uniform a priori estimate. Suppose also that $\Phi: \mathbf{C}^{n}[a, b] \rightarrow Q\left[\mathbf{L}_{1}^{n}[a, b]\right]$ has Property $\mathbf{C}$. Then for any function $v \in \mathbf{L}_{1}^{n}[a, b]$ and any $\varepsilon>0$, there exists a generalized solution $x \in \mathbf{D}^{n}[a, b]$ of the problem (4.1) such that

$$
\|\dot{x}-v\|_{L_{1}^{n}(\mathcal{U})} \leqslant \rho_{\mathbf{L}_{1}^{n}(\mathcal{U})}[v, \overline{\operatorname{dec}} \Phi(x)]+\varepsilon \mu(\mathcal{U})
$$

for any measurable set $\mathcal{U} \subset[a, b]$.

$$
\text { If } \Phi: \mathbf{C}^{n}[a, b] \rightarrow \Omega\left(Q\left[\mathbf{L}_{1}^{n}[a, b]\right]\right) \text {, then the theorem is also valid for } \varepsilon=0 \text {. }
$$

Proof. Let $\Phi: \mathbf{C}^{n}[a, b] \rightarrow Q\left[\mathbf{L}_{1}^{n}[a, b]\right]$ have Property $\mathcal{C}$. Then by Corollary 3.19, the mapping $\widetilde{\Phi}: \mathbf{C}^{n}[a, b] \rightarrow \Pi\left[\mathbf{L}_{1}^{n}[a, b]\right]$ given by (3.25) is continuous. Therefore (see [30-32]), given a number $\varepsilon>0$ and a function $v \in \mathbf{L}_{1}^{n}[a, b]$, there exists a continuous mapping $\varphi: \mathbf{C}^{n}[a, b] \rightarrow \mathbf{L}_{1}^{n}[a, b]$ satisfying $\varphi(y) \in \widetilde{\Phi}(y)$ and

$$
\|\varphi(y)-v\|_{\mathrm{L}_{1}^{n}(\mathcal{U})} \leqslant \rho_{\mathrm{L}_{1}^{n}(\mathcal{U})}[v, \overline{\operatorname{dec}} \Phi(y)]+\varepsilon \mu(\mathcal{U})
$$

for any $y \in \mathbf{C}^{n}[a, b]$ and any measurable set $\mathcal{U} \subset[a, b]$. It follows from Theorem 4.9 that $H\left(x_{0}, \tau\right) \neq \varnothing$ for any $\tau \in(a, b]$, and that there exists a number $r>0$ such that $\|y\|_{C^{n}[a, \tau]} \leqslant r$ for each $\tau \in(a, b], y \in H\left(x_{0}, \tau\right)$. Now, we show that there exists $x \in H\left(x_{0}, b\right)$ satisfying (4.18). Consider the problem

$$
\dot{x} \in \overline{\operatorname{dec}} \Phi\left(W_{r}(x)\right), \quad x(a)=x_{0} \quad\left(x_{0} \in \mathbb{R}^{n}\right),
$$


where the continuous mapping $W_{r}: \mathbf{C}^{n}[a, b] \rightarrow \mathbf{C}^{n}[a, b]$ is given by

$$
\left(W_{r} x\right)(t)= \begin{cases}x(t) & \text { if }|x(t)| \leqslant r+2 \\ \frac{r+2}{|x(t)|} x(t) & \text { if }|x(t)|>r+2 .\end{cases}
$$

We denote by $H(W)$ the set of all solutions of the problem (4.20). Let us show that $H(W)=$ $H\left(x_{0}, b\right)$. It follows from the definition of the mapping $W_{r}: \mathbf{C}^{n}[a, b] \rightarrow \mathbf{C}^{n}[a, b]$ (see (4.21)) that $H\left(x_{0}, b\right) \subset H(W)$. Let us prove that $H(W) \subset H\left(x_{0}, b\right)$. Assume the converse. Then there exists $y \in H(W)$ such that $\|y\|_{\mathrm{C}^{n}[a, b]}>r+2$. Since $y(a)=x_{0}$, we have $|y(a)|<r+2$. This implies that there exists a number $\tau \in(a, b]$ such that $\left\|\left.y\right|_{\tau}\right\|_{\mathrm{C}^{n}[a, \tau]}=r+1\left(\left.y\right|_{\tau}\right.$ is the restriction of the function $y$ to $[a, \tau])$. By $(4.21)$, we have $\left.y\right|_{\tau} \in H\left(x_{0}, \tau\right)$. This contradicts to the definition of the number $r$. Hence, $H\left(x_{0}, b\right)=H(W)$. Consider a continuous operator $\Psi: \mathbf{C}^{n}[a, b] \rightarrow \mathbf{C}^{n}[a, b]$ given by

$$
\Psi(x)=x_{0}+\Lambda \varphi\left(W_{r}(x)\right),
$$

where the operator $\Lambda: \mathbf{L}_{1}^{n}[a, b] \rightarrow \mathbf{C}^{n}[a, b]$ is the operator of integration defined by (4.17), and $\varphi: \mathbf{C}^{n}[a, b] \rightarrow \mathbf{L}_{1}^{n}[a, b]$ is a continuous selection of the mapping $\widetilde{\Phi}: \mathbf{C}^{n}[a, b] \rightarrow \Pi\left[\mathbf{L}_{1}^{n}[a, b]\right]$ given by (3.25). The function $\varphi$ ia also assumed to satisfy (4.19). Since the operator $W_{r}$ : $\mathbf{C}^{n}[a, b] \rightarrow \mathbf{C}^{n}[a, b]$ is bounded, we obtain that the image $\Psi\left(\mathbf{C}^{n}[a, b]\right)$ is a relatively compact subset of $\mathbf{C}^{n}[a, b]$. Hence, the set $U=\overline{\mathrm{co}} \Psi\left(\mathbf{C}^{n}[a, b]\right)$ is a convex compact set. Since the operator $\Psi: \mathbf{C}^{n}[a, b] \rightarrow \mathbf{C}^{n}[a, b]$ given by (4.22) takes the set $U$ into itself, we have, by Schauder theorem, that the mapping $\Psi(\cdot)$ has a fixed point. This fixed point $x$ is the solution of the problem (4.20). It follows from the above equality $H(W)=H\left(x_{0}, b\right)$ that this solution $x \in H(W)$ is a generalized solution of the problem (4.1). Since $\dot{x}=\varphi(x)$, we see that (4.19) implies (4.18).

Let us prove the second statement of the theorem. Let $\Phi: \mathbf{C}^{n}[a, b] \rightarrow \Omega\left(Q\left[\mathbf{L}_{1}^{n}[a, b]\right]\right)$. Suppose also that $\Phi$ has Property $\mathcal{C}$. Then by Lemma 3.9, $\widetilde{\Phi}: \mathbf{C}^{n}[a, b] \rightarrow \Omega\left(\Pi\left[\mathbf{L}_{1}^{n}[a, b]\right]\right)$. Hence for each $i=1,2, \ldots$, there exists a generalized solution $x_{i} \in \mathbf{D}^{n}[a, b]$ of the problem (4.1) such that for any measurable set $\mathcal{U} \subset[a, b]$, the inequality (4.18) is valid for $\dot{x}=\dot{x}_{i}$ and $\varepsilon=1 / i$. Since the set $H\left(x_{0}, b\right)$ is bounded, we see that the sequence $\left\{\dot{x}_{i}\right\}$ is weakly compact in $\mathbf{L}_{1}^{n}[a, b]$. Without loss of generality, it can be assumed that $\dot{x}_{i} \rightarrow \dot{x}$ weakly in $\mathbf{L}_{1}^{n}[a, b]$ and $x_{i} \rightarrow x$ in $\mathrm{C}^{n}[a, b]$ as $i \rightarrow \infty$. Let us show that $x$ is a generalized solution of the problem (4.1). In other words, we have to prove that $\dot{x} \in \overline{\operatorname{dec}} \Phi(x)$. Assume that the functions $y_{i} \in \overline{\operatorname{dec}} \Phi(x), i=$ $1,2, \ldots$, satisfy

$$
\left\|y_{i}-\dot{x}_{i}\right\|_{\mathbf{L}_{1}^{n}[a, b]}=\rho_{\mathbf{L}_{1}^{n}[a, b]}\left[\dot{x}_{i} ; \overline{\operatorname{dec}} \Phi(x)\right]
$$

(as $\overline{\operatorname{dec}} \Phi(x) \in \Pi\left[\mathbf{L}_{1}^{n}[a, b]\right]$, these functions do exist). It follows from (4.23) that

$$
\left\|y_{i}-\dot{x}_{i}\right\|_{\mathrm{L}_{1}^{n}[a, b]} \leqslant h_{\mathrm{L}_{1}^{n}[a, b]}\left[\overline{\operatorname{dec}} \Phi\left(x_{i}\right) ; \overline{\operatorname{dec}} \Phi(x)\right] .
$$

Since the mapping $\widetilde{\Phi}: \mathbf{C}^{n}[a, b] \rightarrow \Omega\left(\Pi\left[\mathbf{L}_{1}^{n}[a, b]\right]\right)$ given by (3.25) is continuous, we obtain, by (4.24), that $y_{i}-\dot{x}_{i} \rightarrow 0$ in $\mathbf{L}_{1}^{n}[a, b]$ as $i \rightarrow \infty$. Since $\dot{x}_{i} \rightarrow \dot{x}$ weakly in $\mathbf{L}_{1}^{n}[a, b]$ as $i \rightarrow \infty$, we have that $y_{i} \rightarrow \dot{x}$ weakly in $\mathbf{L}_{1}^{n}[a, b]$ as $i \rightarrow \infty$. Therefore, the convexity of the set $\overline{\operatorname{dec}} \Phi(x)$ implies that $\dot{x} \in \overline{\operatorname{dec}} \Phi(x)$ (see [21]). Thus, $x$ is a generalized solution of the problem (4.1). 
Further, let us show that (4.19) holds for the solution $x$ and for $\varepsilon=0$. Since $\dot{x}_{i} \rightarrow \dot{x}$ weakly in $\mathbf{L}_{1}^{n}[a, b]$ as $i \rightarrow \infty$, we have, by [21], that for each $m=1,2, \ldots$, there exist numbers $i(m), \lambda_{j}^{m} \geqslant 0, j=1,2, \ldots, i(m)$, satisfying the following conditions: $\sum_{j=1}^{i(m)} \lambda_{j}^{m}=1$; the sequence $\left\{\beta_{m}=\sum_{j=1}^{i(m)} \lambda_{j}^{m} \dot{x}_{j+m}\right\}$ tends to $\dot{x}$ in $\mathbf{L}_{1}^{n}[a, b]$. Since

$$
\|\dot{x}-v\|_{\mathbf{L}_{1}^{n}[a, b]} \leqslant\left\|\dot{x}-\beta_{m}\right\|_{\mathbf{L}_{1}^{n}[a, b]}+\sum_{j=1}^{i(m)} \lambda_{j}^{m}\left\|\dot{x}_{j+m}-v\right\|_{\mathbf{L}_{1}^{n}[a, b]}
$$

for each $m=1,2, \ldots$, it follows, due to the choice of the sequence $\left\{\dot{x}_{i}\right\}$, that

$$
\|\dot{x}-v\|_{\mathbf{L}_{1}^{n}[a, b]} \leqslant\left\|\dot{x}-\beta_{m}\right\|_{\mathbf{L}_{1}^{n}[a, b]}+\sum_{j=1}^{i(m)} \lambda_{j}^{m} \rho_{\mathbf{L}_{1}^{n}[a, b]}\left[v ; \overline{\operatorname{dec}} \Phi\left(x_{j+m}\right)\right]+(b-a) \sum_{j=1}^{i(m)} \lambda_{j}^{m} \frac{1}{j+m}
$$

for each $m=1,2, \ldots$

Since

$$
\lim _{i \rightarrow \infty} \rho_{\mathbf{L}_{1}^{n}[a, b]}\left[v ; \overline{\operatorname{dec}} \Phi\left(x_{i}\right)\right]=\rho_{\mathbf{L}_{1}^{n}[a, b]}[v ; \overline{\operatorname{dec}} \Phi(x)]
$$

it follows that letting $m \rightarrow \infty$ in the previous inequality, we obtain

$$
\|\dot{x}-v\|_{\mathbf{L}_{1}^{n}[a, b]}=\rho_{\mathbf{L}_{1}^{n}[a, b]}[v ; \overline{\operatorname{dec}} \Phi(x)] .
$$

Finally, note that by the decomposability of the set $\overline{\operatorname{dec}} \Phi(x)$, this equality holds for any measurable set $\mathcal{U} \subset[a, b]$. This completes the proof.

Theorems 4.12 and 4.13 yield the following result.

Corollary 4.14. Suppose that a mapping $\Phi: \mathbf{C}^{n}[a, b] \rightarrow Q\left[\mathbf{L}_{1}^{n}[a, b]\right]$ has Properties $\Gamma_{1}$ and $\mathcal{C}$. Then for any function $v \in \mathbf{L}_{1}^{n}[a, b]$ and any $\varepsilon>0$, there exists a generalized solution $x \in \mathbf{D}^{n}[a, b]$ of the problem (4.1) such that (4.18) holds for any measurable set $u \subset[a, b]$.

If $\Phi: \mathbf{C}^{n}[a, b] \rightarrow \Omega\left(Q\left[\mathbf{L}_{1}^{n}[a, b]\right]\right)$, then the corollary is also valid for $\varepsilon=0$.

Remark 4.15. Consider the convex compact set $U=\overline{\mathrm{Co}} \widetilde{\Psi}\left(\mathbf{C}^{n}[a, b]\right) \subset \mathbf{C}^{n}[a, b]$, where the mapping $\widetilde{\Psi}: \mathbf{C}^{n}[a, b] \rightarrow 2^{C^{n}[a, b]}$ is given by

$$
\widetilde{\Psi}(x)=x_{0}+\Lambda \widetilde{\Phi}\left(W_{r}(x)\right)
$$

Here, the operators $\widetilde{\Phi}: \mathbf{C}^{n}[a, b] \rightarrow \Pi\left[\mathbf{L}_{1}^{n}[a, b]\right]$ and $W_{r}: \mathbf{C}^{n}[a, b] \rightarrow \mathbf{C}^{n}[a, b]$ are determined by (3.25) and (4.21), respectively. If a number $r>0$ is such that $\|y\|_{C^{n}[a, \tau]} \leqslant r$ for any $\tau \in(a, b]$, $y \in H\left(x_{0}, \tau\right)$, then due to the the coincidence of the sets $H(W)$ and $H\left(x_{0}, b\right)$ (see the proof of Theorem 4.13), $H\left(x_{0}, b\right) \subset U$.

Definition 4.16. Given $\varepsilon \geq 0, p \geq 0, u \in \mathbf{L}_{+}^{1}[a, b]$, one says that a mapping $\Phi: \mathbf{C}^{n}[a, b] \rightarrow$ $Q\left[\mathbf{L}_{1}^{n}[a, b]\right]$ has Property $\Gamma_{2}^{u, \varepsilon, p}$ if there exists an isotonic and continuous Volterra operator $\Gamma_{2}$ : $\mathbf{C}_{+}^{1}[a, b] \rightarrow \mathbf{L}_{+}^{1}[a, b]$ satisfying the following conditions: 
(i) $\Gamma_{2}(0)=0$;

(ii) for any functions $x, y \in \mathbf{C}^{n}[a, b]$ and any measurable set $\mathcal{U} \subset[a, b]$, one has

$$
h_{\mathrm{L}_{1}^{n}(\mathcal{U})}[\Phi(x) ; \Phi(y)] \leqslant\left\|\Gamma_{2}(Z(x-y))\right\|_{\mathbf{L}_{1}^{1}(\mathcal{U})^{\prime}}
$$

where the continuous mapping $Z: \mathbf{C}^{n}[a, b] \rightarrow \mathrm{C}_{+}^{1}[a, b]$ is determined by (4.10);

(iii) the set of all local solutions of the problem

$$
\dot{y}=u+\varepsilon+\Gamma_{2}(y), \quad y(a)=p,
$$

admits a uniform a priori estimate.

Given $y \in \mathbf{D}^{n}[a, b]$ and $\varkappa \in \mathbf{L}_{+}^{1}[a, b]$, the following estimate will be used in the sequel:

$$
\rho_{\mathbf{L}_{1}^{n}(\mathcal{U})}[\dot{y} ; \Phi(y)] \leqslant \int_{\mathcal{U}} \varkappa(s) d s
$$

for each measurable set $\mathcal{U} \subset[a, b]$.

Theorem 4.17. Let functions $y \in \mathbf{D}^{n}[a, b]$ and $\varkappa \in \mathbf{L}_{+}^{1}[a, b]$ satisfy the inequality (4.32) for each measurable set $U \subset[a, b]$. Suppose that a mapping $\Phi: \mathbf{C}^{n}[a, b] \rightarrow Q\left[\mathbf{L}_{1}^{n}[a, b]\right]$ has Property $\Gamma_{2}^{\varkappa, \varepsilon, p}$, where $\varepsilon \geqslant 0, p=\left|x_{0}-y(a)\right|$, and $x_{0}$ is the initial condition of the problem (4.1). Then for any generalized solution of the problem (4.1) satisfying

$$
\|\dot{x}-\dot{y}\|_{\mathbf{L}_{1}^{n}(\mathcal{U})} \leqslant \rho_{\mathbf{L}_{1}^{n}(\mathcal{U})}[\dot{y} ; \overline{\operatorname{dec}} \Phi(x)]+\varepsilon \mu(\mathcal{U})
$$

for any measurable set $\mathcal{U} \subset[a, b]$, the following conditions are satisfied:

(1)

$$
\Theta(x-y)(t) \leqslant \xi(\varkappa, \varepsilon, p)(t)
$$

for each $t \in[a, b]$, where the function $\xi(\varkappa, \varepsilon, p) \in \mathbf{D}^{1}[a, b]$ is the upper solution of the problem (4.31) for $u=\varkappa$ and $p=\left|x_{0}-y(a)\right|$, and the mapping $\Theta: \mathbf{D}^{n}[a, b] \rightarrow \mathbf{C}_{+}^{1}[a, b]$ is given by (4.15);

$$
|\dot{x}(t)-\dot{y}(t)| \leqslant \varkappa(t)+\varepsilon+\left(\Gamma_{2}(\xi(\varkappa, \varepsilon, p))\right)(t)
$$

for almost all $t \in[a, b]$.

Proof. First, note that since the mapping $\Phi: \mathbf{C}^{n}[a, b] \rightarrow Q\left[\mathbf{L}_{1}^{n}[a, b]\right]$ has Property $\Gamma_{2}^{\varkappa, \varepsilon, p}$, it follows from Theorem 3.18 that so does the mapping $\widetilde{\Phi}: \mathbf{C}^{n}[a, b] \rightarrow \Pi\left[\mathbf{L}_{1}^{n}[a, b]\right]$ determined by (3.25). Further, the inequality (4.33) yields that

$$
\|\dot{x}-\dot{y}\|_{\mathrm{L}_{1}^{n}(\mathcal{U})} \leqslant \rho_{\mathrm{L}_{1}^{n}(\mathcal{U})}[\dot{y} ; \overline{\operatorname{dec}} \Phi(y)]+h_{\mathrm{L}_{1}^{n}(\mathcal{U})}[\overline{\operatorname{dec}} \Phi(y) ; \overline{\operatorname{dec}} \Phi(x)]+\varepsilon \mu(\mathcal{U})
$$

for any measurable set $\mathcal{U} \subset[a, b]$. 
Remark 4.15 and relations (4.36), (4.32), and (4.30) imply that for any measurable set $u \subset[a, b]$, we obtain the inequality

$$
\|\dot{x}-\dot{y}\|_{L_{1}^{n}(\mathcal{U})} \leqslant \int_{\mathcal{U}}\left(\varkappa(s)+\varepsilon+\Gamma_{2}(Z(x-y))(s)\right) d s,
$$

where the mapping $Z: \mathbf{C}^{n}[a, b] \rightarrow \mathbf{C}_{+}^{1}[a, b]$ is given by (4.10). It follows from this inequality that

$$
|\dot{x}(t)-\dot{y}(t)| \leqslant \varkappa(t)+\varepsilon+\Gamma_{2}(Z(x-y))(t)
$$

for almost all $t \in[a, b]$. Since $Z(x-y)(t) \leqslant \Theta(x-y)(t)$ for all $t \in[a, b]$ (see (4.10), (4.15)) and the operator $\left.\Gamma_{2}: \mathbf{C}_{+}^{1}[a, b] \rightarrow \mathbf{L}_{+}^{1}[a, b]\right]$ (see (4.38)) is isotonic, we have that

$$
|\dot{x}(t)-\dot{y}(t)|=\dot{\Theta}(x-y)(t) \leqslant \varkappa(t)+\varepsilon+\Gamma_{2}(\Theta(x-y))(t)
$$

for almost all $t \in[a, b]$. Therefore, (4.39) and the theorem on differential inequalities with an isotonic operator (see [29]) imply (4.34) for any $t \in[a, b]$. The inequality (4.35) follows from (4.34) and (4.39). The proof is complete.

Theorems 4.13 and 4.17 yield the following result.

Theorem 4.18. Let functions $y \in \mathbf{D}^{n}[a, b]$ and $\varkappa \in \mathbf{L}_{+}^{1}[a, b]$ satisfy (4.32) for each measurable set $u \subset[a, b]$. Suppose that a mapping $\Phi: \mathbf{C}^{n}[a, b] \rightarrow Q\left[\mathbf{L}_{1}^{n}[a, b]\right]$ has Property $\Gamma_{2}^{\varkappa, \varepsilon, p}$, where $\varepsilon \geq 0$, $p=\left|x_{0}-y(a)\right|, x_{0}$ is the initial condition in the problem (4.1). Let the set of all local generalized solutions of the problem (4.1) admit a uniform a priori estimate. Then for $\varepsilon>0$, there exists a generalized solution $x \in \mathbf{D}^{n}[a, b]$ of the problem (4.1) which satisfies (4.34) and (4.35) for all $t \in[a, b]$ and for almost all $t \in[a, b]$, respectively.

$$
\text { If } \Phi: \mathbf{C}^{n}[a, b] \rightarrow \Omega\left(Q\left[\mathbf{L}_{1}^{n}[a, b]\right]\right) \text {, then the theorem is also valid for } \varepsilon=0 \text {. }
$$

Corollary 4.19. Let functions $y \in \mathbf{D}^{n}[a, b]$ and $\varkappa \in \mathbf{L}_{+}^{1}[a, b]$ satisfy (4.32) for each measurable set $u \subset[a, b]$. Suppose that a mapping $\Phi: \mathbf{C}^{n}[a, b] \rightarrow Q\left[\mathbf{L}_{1}^{n}[a, b]\right]$ has properties $\Gamma_{1}$ and $\Gamma_{2}^{\varkappa, \varepsilon, p}$, where $\varepsilon \geq 0, p=\left|x_{0}-y(a)\right|, x_{0}$ is the initial condition in the problem (4.1). Then for $\varepsilon>0$, there exists a generalized solution $x \in \mathbf{D}^{n}[a, b]$ of the problem (4.1) which satisfies (4.34) and (4.35) for all $t \in[a, b]$ and for almost all $t \in[a, b]$, respectively.

If $\Phi: \mathbf{C}^{n}[a, b] \rightarrow \Omega\left(Q\left[\mathbf{L}_{1}^{n}[a, b]\right]\right)$, then the corollary is also valid for $\varepsilon=0$.

Remark 4.20. It follows from the proof of Theorem 4.17 that Theorems 4.17, 4.18, and Corollary 4.19 are also valid if the functions $y \in \mathbf{D}^{n}[a, b]$ and $\varkappa \in \mathbf{L}_{+}^{1}[a, b]$ satisfy

$$
\rho_{\mathbf{L}_{1}^{n}(\mathfrak{U})}[\dot{y} ; \overline{\operatorname{dec}} \Phi(y)] \leqslant \int_{\mathcal{U}} \varkappa(s) d s
$$

for each measurable set $\mathcal{U} \subset[a, b]$.

Definition 4.21. An absolutely continuous function $x \in \mathbf{D}^{n}[a, b]$ is called a generalized quasisolution of the problem (4.1) if there exists a sequence of functions $x_{i} \in \mathbf{D}^{n}[a, b], i=1,2, \ldots$, such that the following conditions hold: 
(i) $x_{i} \rightarrow x$ in $\mathrm{C}^{n}[a, b]$ as $i \rightarrow \infty$;

(ii) $\dot{x}_{i} \in \overline{\operatorname{dec}} \Phi(x)$ and $x_{i}(a)=x_{0}$ for each $i=1,2, \ldots$

Note that by Lemma 3.7, if the set $\Phi(x)$ mentioned in Definition 4.21 is decomposable, then a generalized quasisolution coincides with a quasisolution defined in $[9,33]$, where $\Phi(\cdot)$ is the Nemytskii operator. Note also that this definition of a generalized quasisolution differs from the definition of a quasitrajectory given in $[9,33,34]$ due to the condition $\dot{x}_{i} \in \overline{\operatorname{dec}} \Phi(x)$. Using Definition 4.21, we can obtain more general results on the properties of quasisolutions (see Remark 4.23). Moreover, this definition is more suitable for applications.

Let $\mathscr{H}\left(x_{0}\right)$ be the set of all generalized quasisolutions of the problem (4.1).

We define a mapping $\widetilde{\Phi}_{\mathrm{co}}: \mathbf{C}^{n}[a, b] \rightarrow \Omega\left(\Pi\left[\mathbf{L}_{1}^{n}[a, b]\right]\right)$ by the formula

$$
\widetilde{\Phi}_{\mathrm{co}}(x)=\overline{\mathrm{co}}(\overline{\operatorname{dec}} \Phi(x)) \text {. }
$$

We call $\widetilde{\Phi}_{\mathrm{co}}: \mathbf{C}^{n}[a, b] \rightarrow \Omega\left(\Pi\left[\mathbf{L}_{1}^{n}[a, b]\right]\right)$ the convex decomposable hull.

Consider the problem (4.1) with the convex decomposable hull $\widetilde{\Phi}_{\mathrm{co}}: \mathrm{C}^{n}[a, b] \rightarrow$ $\Omega\left(\Pi\left[\mathbf{L}_{1}^{n}[a, b]\right]\right)$ given by (4.41) leading to

$$
\dot{x} \in \widetilde{\Phi}_{\mathrm{co}}(x), \quad x(a)=x_{0} \quad\left(x_{0} \in \mathbb{R}^{n}\right) .
$$

Let $H_{\mathrm{co}}\left(x_{0}, \tau\right)$ be the set of all solutions of the problem (4.42) on the interval $[a, \tau](\tau \in$ $(a, b])$.

Theorem 4.22. $\mathscr{d}\left(x_{0}\right)=H_{\mathrm{co}}\left(x_{0}, b\right)$.

Proof. First, we will show that $H_{\mathrm{co}}\left(x_{0}, b\right) \subset \mathscr{H}\left(x_{0}\right)$. Let $x \in H_{\mathrm{co}}\left(x_{0}, b\right)$. By [35], for $\dot{x} \in$ $\mathbf{L}_{1}^{n}[a, b]$, there exists a sequence $y_{i} \in \overline{\operatorname{dec}} \Phi(x), i=1,2, \ldots$, such that $y_{i} \rightarrow \dot{x}$ weakly in $\mathbf{L}_{1}^{n}[a, b]$ as $i \rightarrow \infty$. This implies that $x_{i}=x_{0}+\Lambda y_{i} \rightarrow x$ in $\mathbf{C}^{n}[a, b]$ as $i \rightarrow \infty$, where $\Lambda: \mathbf{L}_{1}^{n}[a, b] \rightarrow \mathbf{C}^{n}[a, b]$ is the operator of integration (see (4.17)). Hence, $H_{\mathrm{co}}\left(x_{0}, b\right) \subset \mathscr{H}\left(x_{0}\right)$.

Let us now prove that $\mathscr{\ell}\left(x_{0}\right) \subset H_{\mathrm{co}}\left(x_{0}, b\right)$. Let $x \in \mathscr{H}\left(x_{0}\right)$. Then there exists a sequence $x_{i} \in \mathrm{D}^{n}[a, b], i=1,2, \ldots$, satisfying the following conditions: (1) $\dot{x}_{i} \in \overline{\operatorname{dec}} \Phi(x)$ (see (4.41)) and $x_{i}(a)=x_{0}$ for each $i=1,2, \ldots ;(2) x_{i} \rightarrow x$ in $\mathrm{C}^{n}[a, b]$ as $i \rightarrow \infty$. Since the sequence $\dot{x}_{i}$, $i=1,2, \ldots$, is weakly compact, we can assume without loss of generality that $\dot{x}_{i} \rightarrow \dot{x}$ weakly in $\mathbf{L}_{1}^{n}[a, b]$ as $i \rightarrow \infty$. Since $\dot{x}_{i} \in \widetilde{\Phi}_{\text {co }}(x)$ (see (4.41)), it follows that $\dot{x} \in \widetilde{\Phi}_{\mathrm{co}}(x)$ (see [21]). Hence $x \in H_{\mathrm{co}}\left(x_{0}, b\right)$ and therefore $\mathscr{H}\left(x_{0}\right) \subset H_{\mathrm{co}}\left(x_{0}, b\right)$.

Remark 4.23. Theorem 4.22 may still remain valid even if the mapping $\Phi: \mathbf{C}^{n}[a, b] \rightarrow$ $Q\left[\mathbf{L}_{1}^{n}[a, b]\right]$ is discontinuous and its image $\Phi(\mathbb{B})$ is not integrally bounded for every bounded set $\mathbb{B} \subset C^{n}[a, b]$. The proof of Theorem 4.22 is only based on the fact that every value of this mapping is integrally bounded, rather than on the assumption that $\Phi$ is a Volterra operator.

Definition 4.24. One says that a compact convex set $U \subset \mathbf{C}^{n}[a, b]$ has Property $\Phi$ if $\mathscr{H}\left(x_{0}\right) \subset U$, and for any $x \in \mathscr{\ell}\left(x_{0}\right)$, there exists a sequence of absolutely continuous functions $x_{i}:[a, b] \rightarrow$ $\mathbb{R}^{n}, i=1,2, \ldots$, such that

(i) $x_{i} \rightarrow x$ in $\mathbf{C}^{n}[a, b]$ as $i \rightarrow \infty$;

(ii) $x_{i} \in U, \dot{x}_{i} \in \overline{\operatorname{dec}} \Phi(x)$ and $x_{i}(a)=x_{0}$ for each $i=1,2, \ldots$ 
Lemma 4.25. Suppose that the set of all local solutions of the problem (4.41) admits a uniform a priori estimate. Then, there exists a set $U \subset \mathbf{C}^{n}[a, b]$ satisfying Property $\Phi$.

Proof. It follows from Theorem 4.22 and Remark 4.15 that the set $U=\overline{\operatorname{co}} \widetilde{\Psi}\left(\mathbf{C}^{n}[a, b]\right)$ has Property $\boldsymbol{\Phi}$. Here, the mapping $\widetilde{\Psi}: C^{n}[a, b] \rightarrow 2^{C^{n}[a, b]}$ is determined by $(4.29)$, where $\widetilde{\Phi}(\cdot) \equiv$ $\widetilde{\Phi}_{\mathrm{co}}(\cdot)$.

Lemma 4.26. Let sets $\Phi_{i} \in \Pi\left[\mathbf{L}_{1}^{n}[a, b]\right], i=1,2$, satisfy $\Phi_{i}=S\left(F_{i}(\cdot)\right), i=1,2$, where $F_{i}:[a, b] \rightarrow$ comp $\left[\mathbb{R}^{n}\right]$ are measurable mappings. Then for any measurable set $\mathcal{U} \subset[a, b]$, one has

$$
h_{\mathbf{L}_{1}^{n}(\mathcal{U})}\left[\Phi_{1} ; \Phi_{2}\right] \leqslant \int_{\mathcal{U}} h\left[F_{1}(t) ; F_{2}(t)\right] d t \leqslant 2 h_{\mathbf{L}_{1}^{n}(\mathcal{U})}\left[\Phi_{1} ; \Phi_{2}\right]
$$

Proof. Let $u \subset[a, b]$ be a measurable set. Put

$$
\tilde{u}=\left\{t \in \mathcal{U}: h^{+}\left[F_{1}(t) ; F_{2}(t)\right] \geq h^{+}\left[F_{2}(t) ; F_{1}(t)\right]\right\} .
$$

The set $\tilde{\mathfrak{U}} \subset \boldsymbol{U}$ is measurable. Since

$$
\int_{\mathcal{U}} h\left[F_{1}(t) ; F_{2}(t)\right] d t=\int_{\tilde{\mathcal{U}}} h^{+}\left[F_{1}(t) ; F_{2}(t)\right] d t+\int_{\mathcal{U} \tilde{\mathcal{U}}} h^{+}\left[F_{2}(t) ; F_{1}(t)\right] d t
$$

we have

$$
\int_{\mathcal{U}} h\left[F_{1}(t) ; F_{2}(t)\right] d t=h_{\mathrm{L}_{1}^{n}(\tilde{\mathcal{u}})}^{+}\left[\Phi_{1} ; \Phi_{2}\right]+h_{\left.\mathrm{L}_{1}^{n}(\mathcal{U}) \tilde{\mathcal{u}}\right)}^{+}\left[\Phi_{2} ; \Phi_{1}\right]
$$

This implies (4.43), and the proof is completed.

Let $F:[a, b] \rightarrow \operatorname{comp}\left[\mathbb{R}^{n}\right]$ be a measurable mapping. Let a mapping co $F:[a, b] \rightarrow$ comp $\left[\mathbb{R}^{n}\right]$ be defined by

$$
(\operatorname{co} F)(t)=\operatorname{co}(F(t)) .
$$

Corollary 4.27. Let sets $\Phi_{i} \in \Pi\left[\mathbf{L}_{1}^{n}[a, b]\right], i=1,2$, satisfy $\Phi_{i}=S\left(F_{i}(\cdot)\right), i=1,2$, where $F_{i}:[a, b] \rightarrow$ comp $\left[\mathbb{R}^{n}\right]$ are measurable mappings. Then for any measurable set $\mathcal{U} \subset[a, b]$, one has

$$
h_{\mathrm{L}_{1}^{n}(\mathcal{U})}\left[\overline{\mathrm{CO}}\left(\Phi_{1}\right) ; \overline{\mathrm{CO}}\left(\Phi_{2}\right)\right] \leqslant 2 h_{\mathrm{L}_{1}^{n}(\mathcal{U})}\left[\Phi_{1} ; \Phi_{2}\right] .
$$

Proof. By [35], we have that $\overline{\mathrm{CO}}\left(\Phi_{i}\right)=S\left(\operatorname{co} F_{i}(\cdot)\right), i=1,2$. Therefore,

$$
h_{\mathrm{L}_{1}^{n}(\mathcal{U})}\left[\overline{\mathrm{CO}}\left(\Phi_{1}\right) ; \overline{\mathrm{CO}}\left(\Phi_{2}\right)\right] \leqslant \int_{\mathcal{U}} h\left[\left(\mathrm{co}_{1}\right)(t) ;\left(\mathrm{co}_{2}\right)(t)\right] d t
$$

for any measurable set $\mathcal{U} \subset[a, b]$. Since, for any measurable set $\mathcal{U} \subset[a, b]$,

$$
\int_{\mathcal{U}} h\left[\left(\operatorname{co} F_{1}\right)(t) ;\left(\operatorname{co} F_{2}\right)(t)\right] d t \leqslant \int_{\mathcal{U}} h\left[\left(F_{1}\right)(t) ;\left(F_{2}\right)(t)\right] d t,
$$

we obtain, due to (4.43), the inequality (4.48). 
Definition 4.28. One says that a mapping $\Phi: \mathbf{C}^{n}[a, b] \rightarrow Q\left[\mathbf{L}_{1}^{n}[a, b]\right]$ has Property $\Gamma_{3}$ if Property $\Gamma_{2}^{0,0,0}$ is satisfied and the following conditions hold:

(i) $\Gamma_{2}(0)=0$;

(ii) on every interval $[a, \tau](\tau \in(a, b])$, there exists a unique zero solution of the problem (4.31), where $u=0, \varepsilon=0, p=0$.

Theorem 4.29. Suppose that the set of all local generalized solutions of the problem (4.1) admits a uniform a priory estimate. Suppose also that a mapping $\Phi: \mathbf{C}^{n}[a, b] \rightarrow Q\left[\mathbf{L}_{1}^{n}[a, b]\right]$ satisfies Property $\Gamma_{3}$. Then, $H\left(x_{0}, b\right) \neq \varnothing$ and

$$
\overline{H\left(x_{0}, b\right)}=H_{\mathrm{co}}\left(x_{0}, b\right),
$$

where $\overline{H\left(x_{0}, b\right)}$ is the closure of the set $H\left(x_{0}, b\right)$ in $\mathrm{C}^{n}[a, b]$.

Proof. Let us first prove that the set $H_{\mathrm{co}}\left(x_{0}\right)$ is closed in $\mathrm{C}^{n}[a, b]$. Indeed, suppose that a sequence $x_{i} \in H_{\mathrm{co}}\left(x_{0}\right), i=1,2, \ldots$, tends to $x$ in $\mathrm{C}^{n}[a, b]$ as $i \rightarrow \infty$. Since the sequence $\left\{\dot{x}_{i}\right\}$ is integrally bounded, it follows that $x_{i} \in \mathbf{D}^{n}[a, b]$ and $\dot{x}_{i} \rightarrow \dot{x}$ weakly in $\mathbf{L}_{1}^{n}[a, b]$ as $i \rightarrow \infty$. For each $i=1,2, \ldots$, let the function $z_{i} \in \widetilde{\Phi}_{\mathrm{co}}(x)$ satisfy

$$
\left\|\dot{x}_{i}-z_{i}\right\|_{\mathbf{L}_{1}^{n}[a, b]}=\rho_{\mathbf{L}_{1}^{n}[a, b]}\left[\dot{x}_{i} ; \tilde{\Phi}_{\mathrm{co}}(x)\right]
$$

where $\widetilde{\Phi}_{\mathrm{co}}: \mathrm{C}^{n}[a, b] \rightarrow \Omega\left(\Pi\left[\mathbf{L}_{1}^{n}[a, b]\right]\right)$ is the convex decomposable hull given by (4.41). Since the mapping $\tilde{\Phi}: \mathbf{C}^{n}[a, b] \rightarrow \Pi\left[\mathbf{L}_{1}^{n}[a, b]\right]$ given by (3.25) is Hausdorff continuous, it follows from (4.48) that so is the mapping $\widetilde{\Phi}_{\mathrm{co}}(x): \mathbf{C}^{n}[a, b] \rightarrow \Omega\left(\Pi\left[\mathbf{L}_{1}^{n}[a, b]\right]\right)$. Therefore, (4.52) implies that $\dot{x}_{i}-z_{i} \rightarrow 0$ in $\mathbf{L}_{1}^{n}[a, b]$ as $i \rightarrow \infty$. Hence, $z_{i} \rightarrow \dot{x}$ weakly in $\mathbf{L}_{1}^{n}[a, b]$ as $i \rightarrow \infty$. Since the set $\widetilde{\Phi}_{\mathrm{co}}(x)$ is convex, we have (see [21]) that $\dot{x} \in \widetilde{\Phi}_{\mathrm{co}}(x)$. Therefore, the set $H_{\mathrm{co}}\left(x_{0}\right)$ is closed in $\mathrm{C}^{n}[a, b]$.

Now, let us prove the equality (4.51). The closedness of the set $H_{\mathrm{co}}\left(x_{0}\right)$ yields that $\overline{H\left(x_{0}, b\right)} \subset H_{\mathrm{co}}\left(x_{0}\right)$. Further, let us show that $H_{\mathrm{co}}\left(x_{0}\right) \subset \overline{H\left(x_{0}, b\right)}$. Suppose $x \in H_{\mathrm{co}}\left(x_{0}\right)$. Then from Theorem 4.22, it follows that there exists a sequence $y_{i} \in \mathbf{D}^{n}[a, b], i=1,2, \ldots$, such that $y_{i} \in \tilde{\Phi}(x), y_{i}(a)=x_{0}, i=1,2, \ldots\left(x_{0}\right.$ is the initial condition in the problem (4.1)) and $y_{i} \rightarrow x$ in $\mathbf{C}^{n}[a, b]$ as $i \rightarrow \infty$. Since the mapping $\Phi: \mathbf{C}^{n}[a, b] \rightarrow Q\left[\mathbf{L}_{1}^{n}[a, b]\right]$ has Property $\Gamma_{3}$, we see that, due to (4.30),

$$
\rho_{\mathbf{L}_{1}^{n}(\mathcal{U})}\left[\dot{y}_{i} ; \Phi\left(y_{i}\right)\right] \leqslant h_{\mathbf{L}_{1}^{n}(\mathcal{U})}\left[\Phi(x) ; \Phi\left(y_{i}\right)\right] \leqslant \int_{\mathcal{U}}\left(\Gamma_{2}\left(Z\left(x-y_{i}\right)\right)\right)(s) d s
$$

for each $i=1,2, \ldots$ and any measurable set $U \subset[a, b]$. Here, the operator $Z: \mathbf{C}^{n}[a, b] \rightarrow$ $\mathbf{C}_{+}^{1}[a, b]$ is given by (4.10). Since the mapping $\Gamma_{2}: \mathbf{C}_{+}^{1}[a, b] \rightarrow \mathbf{L}_{+}^{1}[a, b]$ is continuous and $\Gamma_{2}(0)=$ 0 , we have that $\varkappa_{i}=\Gamma_{2}\left(Z\left(x-y_{i}\right)\right) \rightarrow 0$ in $\mathbf{L}_{1}^{1}[a, b]$ as $i \rightarrow \infty$. Since the problem (4.31) with $u=0$, $\varepsilon=0$, and $p=0$ only has the zero solution on each interval $[a, \tau](\tau \in(a, b])$, we see that the set of all local solutions of the problem (4.31) with $u=\varkappa_{i}, \varepsilon=1 / i$, and $p=0$ admits a uniform a priori estimate starting from some $i=1,2, \ldots$ (see [36]). Renumerating, we may assume without loss of generality that this holds true for all $i=1,2, \ldots$. This implies (see [29]) that for each $i=1,2, \ldots$, there exists the upper solution $\xi\left(\varkappa_{i}, 1 / i, 0\right)$ of the problem (4.31) with $u=\varkappa_{i}$, $\varepsilon=1 / i$, and $p=0$. Hence, it follows from Theorem 4.18 that for each $i=1,2, \ldots$, there exists 
a generalized solution $x_{i} \in \mathbf{D}^{n}[a, b]$ of the problem (4.1) satisfying $\Theta\left(x_{i}-y_{i}\right) \leqslant \xi\left(\varkappa_{i}, 1 / i, 0\right)$, where the continuous operator $\Theta: \mathbf{D}^{n}[a, b] \rightarrow \mathbf{C}_{+}^{1}[a, b]$ is given by (4.15). Since $\xi\left(\varkappa_{i}, 1 / i, 0\right) \rightarrow 0$ in $\mathrm{C}^{1}[a, b]$ as $i \rightarrow \infty$, we have that $\Theta\left(x_{i}-y_{i}\right) \rightarrow 0$ as $i \rightarrow \infty$. Since $y_{i} \rightarrow x$ in $C^{n}[a, b]$ as $i \rightarrow \infty$, we see that $x_{i} \rightarrow x$ in $\mathrm{C}^{n}[a, b]$ as $i \rightarrow \infty$. Therefore, $x \in \overline{H\left(x_{0}, b\right)}$ and consequently $H_{\mathrm{co}}\left(x_{0}\right) \subset \overline{H\left(x_{0}, b\right)}$. This yields (4.51). The proof is complete.

Corollary 4.30. Suppose that a mapping $\Phi: \mathbf{C}^{n}[a, b] \rightarrow Q\left[\mathbf{L}_{1}^{n}[a, b]\right]$ has Properties $\Gamma_{1}$ and $\Gamma_{3}$. Then $H\left(x_{0}, b\right) \neq \varnothing$ and the equality (4.51) is satisfied.

Remark 4.31. If the solution set of a differential inclusion with nonconvex multivalued mapping is dense in the solution set of the convexified inclusion, then such a property is called the density principle. The density principle is a fundamental property in the theory of differential inclusions (see [13]). Many papers (e.g., [3, 4, 6, 10-12, 23-25, 29-32, 37-39]) deal with the justification of the density principle. Theorem 4.29 and Corollary 4.30 justify the density principle for the generalized solutions of the problem (4.1).

\section{Generalized approximate solutions of the functional differential equation}

Approximate solutions are of great importance in the study of differential equations and inclusions (see [4, 40-43]). They are used in the theorems on existence (e.g., Euler curves) as well as in the study of the dependence of a solution on initial conditions and the right-hand side of the equation. In $[40,41]$, the definition of an approximate solution of a differential equation with piecewise continuous right-hand side was given, using so-called internal and external perturbations. This definition not only deals with small changes of the right-hand side within its domain of continuity, but also with the small changes in the boundaries of these domains. A more general definition of an approximate solution, which can be used not only for the study of functional differential equations with discontinuous right-hand sides but also for differential inclusions with upper semicontinuous convex right-hand sides, was given in [4]. In this paper, the following important property was justified for such an inclusion: the limit of approximate solutions is again a solution of functional differential inclusion. In the present paper, we introduce various definitions of generalized approximate solutions of a functional differential inclusion. The main difference of our definitions from the one given in [4] is that the values of a multivalued mapping are not convexified. Due to this, the topological properties of the sets of generalized approximate solutions are studied and the density principle is proven.

Since a generalized solution of the problem (4.1) is determined by the closed decomposable hull of a set, it is natural to raise the following question: how robust is the set of the generalized solutions of (4.1) with respect to small perturbations of $\overline{\operatorname{dec}} \Phi(x)$ ? It follows from Remark 3.10 that constructing $\overline{\operatorname{dec}} \Phi(x)$ for each fixed $x \in \mathrm{C}^{n}[a, b]$ is equivalent to finding a measurable, integrally bounded mapping $\Delta_{x}:[a, b] \rightarrow \operatorname{comp}\left[\mathbb{R}^{n}\right]$ satisfying

$$
\overline{\operatorname{dec}} \Phi(x)=S\left(\Delta_{x}(\cdot)\right) \text {. }
$$

The mapping $\Delta_{x}:[a, b] \rightarrow \operatorname{comp}\left[\mathbb{R}^{n}\right]$ is, in the sequel, written as $\Delta:[a, b] \times \mathbf{C}^{n}[a, b] \rightarrow$ comp $\left[\mathbb{R}^{n}\right]$ and called a mapping generating the mapping $\widetilde{\Phi}: \mathbf{C}^{n}[a, b] \rightarrow \Pi\left[\mathbf{L}_{1}^{n}[a, b]\right]$ given by (3.25).

Denote by $K([a, b] \times[0, \infty))$ the set of all continuous functions $\eta:[a, b] \times[0, \infty) \rightarrow[0, \infty)$ satisfying the following conditions: 
(1) for each $\delta \geqslant 0, \eta(\cdot, \delta) \in \mathbf{L}_{1}^{1}[a, b]$;

(2) for each $\delta \geqslant 0$, there exists a function $\beta_{\delta}(\cdot) \in \mathbf{L}_{1}^{1}[a, b]$ such that $\eta(t, \tau) \leqslant \beta_{\delta}(t)$ for almost all $t \in[a, b]$ and all $\tau \in[0, \delta]$;

(3) $\lim _{\delta \rightarrow 0+0} \eta(t, \delta)=\eta(t, 0)=0$ for almost all $t \in[a, b]$.

Since the mappings $\Delta(\cdot, \cdot)$ and $\Phi(\cdot)$ are related by the equality (5.1), we have that the robustness of the set of the generalized solutions of (4.1) with respect to small perturbations of $\overline{\operatorname{dec}} \Phi(x)$ can be studied via the robustness properties of $\Delta$. Assume that the perturbation $\Delta_{\eta}(t, x, \delta)$ (e.g., an error in measurements of $\left.\Delta(t, x)\right)$ is given by

$$
\Delta_{\eta}(t, x, \delta)=(\Delta(t, x))^{\eta(t, \delta)},
$$

where $\eta(\cdot, \cdot) \in K([a, b] \times[0, \infty))$ (here, $(\Delta(t, x))^{\eta(t, \delta)}$ is an $\eta$-neighborhood of the set $\Delta(t, x)$, see Preliminaries).

Note that (5.2) yields

$$
h\left[\Delta(t, x) ; \Delta_{\eta}(t, x, \delta)\right]=\eta(t, \delta)
$$

for all $(t, x) \in[a, b] \times \mathbf{C}^{n}[a, b]$. Thus, (5.3) implies that

$$
\lim _{\delta \rightarrow+0} h\left[\Delta(t, x) ; \Delta_{\eta}(t, x, \delta)\right]=0
$$

for each function $\eta(\cdot, \cdot) \in K([a, b] \times[0, \infty))$, almost all $t \in[a, b]$, and all $x \in \mathbf{C}^{n}[a, b]$. Therefore, all mappings $\Delta_{\eta}:[a, b] \times \mathbf{C}^{n}[a, b] \times[0, \infty) \rightarrow \operatorname{comp}\left[\mathbb{R}^{n}\right]$ defined by (5.2) and depending on $\eta(\cdot, \cdot) \in K([a, b] \times[0, \infty))$ are close (in the sense of (5.4)) to the mapping $\Delta:[a, b] \times \mathbf{C}^{n}[a, b] \rightarrow$ comp $\left[\mathbb{R}^{n}\right]$. The mapping $\Delta_{\eta}:[a, b] \times \mathbf{C}^{n}[a, b] \times[0, \infty) \rightarrow \operatorname{comp}\left[\mathbb{R}^{n}\right]$ is called the approximating operator.

We define a mapping $\widetilde{\Phi}_{\eta}: \mathbf{C}^{n}[a, b] \times[0, \infty) \rightarrow \Pi\left[\mathbf{L}_{1}^{n}[a, b]\right]$ by the formula

$$
\widetilde{\Phi}_{\eta}(x, \delta)=S\left(\Delta_{\eta}(\cdot, x, \delta)\right)
$$

where the operator $\Delta_{\eta}:[a, b] \times \mathrm{C}^{n}[a, b] \times[0, \infty) \rightarrow \operatorname{comp}\left[\mathbb{R}^{n}\right]$ is given by (5.2). The equalities (5.3) and (5.5) imply that

$$
h_{\mathbf{L}_{1}^{n}[a, b]}\left[\widetilde{\Phi}_{\eta}(x, \delta) ; \tilde{\Phi}(x)\right]=\int_{a}^{b} \eta(t, \delta) d t
$$

for any $x \in \mathbf{C}^{n}[a, b]$.

It follows from (5.6) and the Lebesgue theorem that

$$
\lim _{\delta \rightarrow 0+0} h_{\mathbf{L}_{1}^{n}[a, b]}\left[\widetilde{\Phi}_{\eta}(x, \delta) ; \widetilde{\Phi}(x)\right]=0 .
$$

Thus, all mappings $\widetilde{\Phi}_{\eta}: \mathbf{C}^{n}[a, b] \times[0, \infty) \rightarrow \Pi\left[\mathbf{L}_{1}^{n}[a, b]\right]$ defined by (5.2) and (5.5) and depending on $\eta(\cdot, \cdot) \in K([a, b] \times[0, \infty))$ are close (in the sense of (5.7)) to the mapping $\tilde{\Phi}$ : $\mathbf{C}^{n}[a, b] \rightarrow \Pi\left[\mathbf{L}_{1}^{n}[a, b]\right]$ given by (3.25). 
Lemma 5.1 (see [6]). Let $X$ be a normed space and let $U \subset X$ be a convex set. Then

$$
h_{X}\left[B_{X}\left[x_{1}, r_{1}\right] \cap U ; B_{X}\left[x_{2}, r_{2}\right] \cap U\right] \leqslant\left\|x_{1}-x_{2}\right\|_{X}+\left|r_{2}-r_{1}\right|
$$

for all $x_{1}, x_{2} \in U$ and all $r_{1}, r_{2}>0$.

Denote by $P\left(\mathbf{C}^{n}[a, b] \times[0, \infty)\right)$ the set of all continuous functions $\omega: \mathbf{C}^{n}[a, b] \times[0, \infty) \rightarrow$ $[0, \infty)$ such that $\omega(x, 0)=0$ for any $x \in \mathbf{C}^{n}[a, b]$ and $\omega(x, \delta)>0$ for any $(x, \delta) \in \mathbf{C}^{n}[a, b] \times(0, \infty)$.

Let $U \subset \mathbf{C}^{n}[a, b]$ be a closed convex set and let $\omega(\cdot, \cdot) \in P\left(\mathbf{C}^{n}[a, b] \times[0, \infty)\right)$. We define a multivalued mapping $M_{U}(\omega): U \times[0, \infty) \rightarrow \Omega(U)$ by

$$
M_{U}(\omega)(x, \delta)=\overline{B_{\mathrm{C}^{n}[a, b]}[x, \omega(x, \delta)]} \cap U .
$$

The inequality (5.8) yields the following result.

Lemma 5.2. Let $U \subset \mathbf{C}^{n}[a, b]$ be a closed convex set and let $\omega(\cdot, \cdot) \in P\left(\mathbf{C}^{n}[a, b] \times[0, \infty)\right)$. Then, $a$ multivalued mapping $M_{U}(\omega): U \times[0, \infty) \rightarrow \Omega(U)$ given by (5.9) is Hausdorff continuous.

We define a mapping $\varphi_{U}(\omega):[a, b] \times U \times[0, \infty) \rightarrow[0, \infty)$ by the formula

$$
\varphi_{U}(\omega)(t, x, \delta)=\sup _{y \in M_{U}(\omega)(x, \delta)} h[\Delta(t, x) ; \Delta(t, y)]
$$

where the mapping $M_{U}(\omega): U \times[0, \infty) \rightarrow \Omega(U)$ is given by (5.9) and the mapping $\Delta$ : $[a, b] \times \mathbf{C}^{n}[a, b] \rightarrow$ comp $\left[\mathbb{R}^{n}\right]$ generates the mapping $\widetilde{\Phi}$ given by (3.25).

It is natural to address the value of the function $\varphi_{U}(\omega)(\cdot, \cdot, \cdot)$ at the point $(t, x, \delta) \in[a, b] \times$ $U \times[0, \infty)$ as the modulus of continuity of the mapping $\Delta:[a, b] \times C^{n}[a, b] \rightarrow \operatorname{comp}\left[\mathbb{R}^{n}\right]$ at the point $(t, x)$ with respect to the variable $x \in U$. We call the function $\omega(\cdot, \cdot)$ the radius of continuity, while the function $\varphi_{U}(\cdot, \cdot, \cdot)$ itself is called the modulus of continuity of the mapping $\Delta:[a, b] \times \mathbf{C}^{n}[a, b] \rightarrow \operatorname{comp}\left[\mathbb{R}^{n}\right]$ with respect to the radius of continuity $\omega(\cdot, \cdot)$.

Definition 5.3. One says that a mapping $\Phi: \mathbf{C}^{n}[a, b] \rightarrow Q\left[\mathbf{L}^{n}[a, b]\right]$ has Property $\tilde{C}$ if the mapping $\Delta:[a, b] \times \mathbf{C}^{n}[a, b] \rightarrow \operatorname{comp}\left[\mathbb{R}^{n}\right]$ generating the mapping $\widetilde{\Phi}: \mathbf{C}^{n}[a, b] \rightarrow \Pi\left[\mathbf{L}_{1}^{n}[a, b]\right]$ given by (3.25) is Hausdorff continuous in the second variable for almost all $t \in[a, b]$.

Lemma 5.4. Suppose that for a mapping $\Phi: \mathbf{C}^{n}[a, b] \rightarrow Q\left[\mathbf{L}^{n}[a, b]\right]$, there exists an isotonic continuous operator $\Gamma: \mathbf{C}_{+}^{1}[a, b] \rightarrow \mathbf{L}_{+}^{1}[a, b]$ satisfying the following conditions:

(i) $\Gamma(0)=0$;

(ii) the inequality (4.30), where $\Gamma_{2} \equiv \Gamma$, is satisfied for any $x, y \in \mathbf{C}^{n}[a, b]$ and any measurable set $u \subset[a, b]$.

Then the mapping $\Phi(\cdot)$ has Property $\tilde{\mathcal{C}}$.

Proof. Let $x_{i} \rightarrow x$ in $\mathrm{C}^{n}[a, b]$ as $i \rightarrow \infty$. Let us show that

$$
\lim _{i \rightarrow \infty} h\left[\Delta\left(t, x_{i}\right) ; \Delta(t, x)\right]=0
$$

for almost all $t \in[a, b]$. 
For each $i=1,2, \ldots$, put $y_{i}=\sup _{j \geqslant i}\left\|x_{j}-x\right\|_{\mathrm{C}^{n}[a, b]}$. Due to Theorem 3.18, (4.43), and the isotonity of the operator $\Gamma: \mathbf{C}_{+}^{1}[a, b] \rightarrow \mathbf{L}_{+}^{1}[a, b]$, for each $i=1,2, \ldots$ and almost all $t \in[a, b]$, we have

$$
h\left[\Delta\left(t, x_{i}\right) ; \Delta(t, x)\right] \leqslant 2 \Gamma\left(Z\left(x_{i}-x\right)\right)(t) \leqslant 2 \Gamma\left(y_{i}\right)(t) .
$$

Since the sequence $\Gamma\left(y_{i}\right), i=1,2, \ldots$, decreases, we obtain, due to the continuity of the mapping $\Gamma(\cdot)$ and the equality $\Gamma(0)=0$, the equality (5.11). This completes the proof.

Lemma 5.5. Let $U$ be a nonempty, convex, compact set in the space $\mathrm{C}^{n}[a, b]$ and let $\omega(\cdot, \cdot) \in$ $P\left(\mathbf{C}^{n}[a, b] \times[0, \infty)\right)$. Suppose also that a mapping $\Phi: \mathbf{C}^{n}[a, b] \rightarrow Q\left[\mathbf{L}_{1}^{n}[a, b]\right]$ has Property $\widetilde{C}$. Then the mapping $\varphi_{U}(\omega):[a, b] \times U \times[0, \infty)$ given by (5.10) has the following properties:

(i) $\varphi_{U}(\omega)(\cdot, x, \delta)$ is measurable for any $(x, \delta) \in U \times[0, \infty)$;

(ii) $\varphi_{U}(t, \cdot, \cdot)$ is continuous on $U \times[0, \infty)$ for almost all $t \in[a, b]$;

(iii) for any $x \in U$ and for almost all $t \in[a, b]$,

$$
\lim _{z \rightarrow x, \delta \rightarrow 0+0} \varphi_{U}(\omega)(t, x, \delta)=0 ;
$$

(iv) there exists an integrable function $p_{U}:[a, b] \rightarrow[0, \infty)$ such that $\varphi_{U}(\omega)(t, x, \delta) \leqslant p_{U}(t)$ for almost all $t \in[a, b]$, any $x \in U$, and all $\delta \in[0, \infty)$.

Definition 5.6. Let $U \subset \mathbf{C}^{n}[a, b]$. One says that the function $\eta(\cdot, \cdot) \in K([a, b] \times[0, \infty))$ provides on $U$ a uniform with respect to the radius of continuity $\omega(\cdot, \cdot) \in P\left(\mathbf{C}^{n}[a, b] \times[0, \infty)\right)$ estimate from above for the modulus of continuity of the mapping $\Delta:[a, b] \times \mathbf{C}^{n}[a, b] \rightarrow \operatorname{comp}\left[\mathbb{R}^{n}\right]$; if for any $\varepsilon>0$ there exists $\delta(\varepsilon)>0$ such that for almost all $t \in[a, b]$, all $x \in U$, and $\delta \in(0, \delta(\varepsilon)]$, one has

$$
\varphi_{U}(\omega)(t, x, \delta) \leqslant \eta(t, \varepsilon),
$$

where $\varphi_{U}:[a, b] \times U \times[0, \infty) \rightarrow[0, \infty)$ is given by $(5.10)$.

Let $U \subset \mathbf{C}^{n}[a, b]$ and $\omega(\cdot, \cdot) \in P\left(\mathbf{C}^{n}[a, b] \times[0, \infty)\right)$. One defines a function $\lambda_{U}(\omega):[a, b] \times$ $[0, \infty) \rightarrow[0, \infty)$ by

$$
\lambda_{U}(\omega)(t, \delta)=\sup _{x \in U} \varphi_{U}(\omega)(t, x, \delta)
$$

Lemma 5.1 yields the following result.

Corollary 5.7. Let $U$ be a nonempty, convex, compact set in the space $\mathbf{C}^{n}[a, b]$ and let $\omega(\cdot, \cdot) \in$ $P\left(\mathbf{C}^{n}[a, b] \times[0, \infty)\right)$. Suppose also that a mapping $\Phi: \mathbf{C}^{n}[a, b] \rightarrow Q\left[\mathbf{L}_{1}^{n}[a, b]\right]$ has Property $\tilde{\mathbf{C}}$. Then the mapping $\lambda_{U}(\omega):[a, b] \times[0, \infty) \rightarrow[0, \infty)$ given by $(5.15)$ belongs to the set $K([a, b] \times[0, \infty))$ and provides a uniform (in the sense of Definition 5.6) estimate from above for the modulus of continuity of the mapping $\Delta:[a, b] \times \mathrm{C}^{n}[a, b] \rightarrow \operatorname{comp}\left[\mathbb{R}^{n}\right]$.

Remark 5.8. Corollary 5.7 yields that if $U$ is a nonempty, convex, compact set in the space $\mathrm{C}^{n}[a, b]$ and a mapping $\Phi: \mathbf{C}^{n}[a, b] \rightarrow Q\left[\mathbf{L}_{1}^{n}[a, b]\right]$ has Property $\widetilde{C}$, then for a given $\omega(\cdot, \cdot) \in$ $P\left(\mathbf{C}^{n}[a, b] \times[0, \infty)\right)$, there exists at least one function $\eta(\cdot, \cdot) \in K([a, b] \times[0, \infty))$ that provides a uniform (in the sense of Definition 5.6) estimate from above for the modulus of continuity of the mapping $\Delta:[a, b] \times \mathbf{C}^{n}[a, b] \rightarrow \operatorname{comp}\left[\mathbb{R}^{n}\right]$. 
Let $\eta(\cdot, \cdot) \in K([a, b] \times[0, \infty))$. For each $\delta \in[0, \infty)$, consider the initial value problem

$$
\dot{x} \in \tilde{\Phi}_{\eta}(x, \delta), \quad x(a)=x_{0} \quad\left(x_{0} \in \mathbb{R}^{n}\right),
$$

where the mapping $\widetilde{\Phi}_{\eta}: \mathbf{C}^{n}[a, b] \times[0, \infty) \rightarrow \Pi\left[\mathbf{L}_{1}^{n}[a, b]\right]$ is given by (5.1) and (5.5).

Since the operator $\widetilde{\Phi}: \mathbf{C}^{n}[a, b] \rightarrow \Pi\left[\mathbf{L}_{1}^{n}[a, b]\right]$ given by (3.25) is a Volterra operator, we see that the mapping $\Delta:[a, b] \times \mathbf{C}^{n}[a, b] \rightarrow \operatorname{comp}\left[\mathbb{R}^{n}\right]$ has the following property: if $x=y$ on $[a, \tau](\tau \in(a, b])$, then $\Delta(t, x)=\Delta(t, y)$ for almost all $t \in[a, \tau]$. This property, (5.1), and (5.4) imply that the operator $\widetilde{\Phi}_{\eta}: \mathbf{C}^{n}[a, b] \times[0, \infty) \rightarrow \Pi\left[\mathbf{L}_{1}^{n}[a, b]\right]$ is a Volterra operator for each $\delta \in[0, \infty)$.

Any solution of the problem (5.16) with a given $\delta>0$ is said to be a generalized $\delta$ solution (a generalized approximate solution with external perturbations) of the problem (4.1). We denote by $H_{\eta(\delta)}(U)$ the set of all generalized $\delta$-solutions of (4.1) belonging to $U \subset \mathrm{C}^{n}[a, b]$.

Theorem 5.9. Suppose that a set $U \subset \mathbf{C}^{n}[a, b]$ has Property $\Phi$. Then for any function $\eta(\cdot, \cdot) \in$ $K([a, b] \times[0, \infty)$ ) that provides a uniform (in the sense of Definition 5.6) estimate from above for the modulus of continuity of the mapping $\Delta:[a, b] \times \mathbf{C}^{n}[a, b] \rightarrow \operatorname{comp}\left[\mathbb{R}^{n}\right]$, one has

$$
H_{\mathrm{co}}\left(x_{0}, b\right)=\bigcap_{\delta>0} \overline{H_{\eta(\delta)}(U)}
$$

where $\overline{H_{\eta(\delta)}(U)}$ is the closure of $H_{\eta(\delta)}(U)$ in $\mathrm{C}^{n}[a, b]$.

Proof. First, let us prove that

$$
H_{\mathrm{co}}\left(x_{0}, b\right) \subset \bigcap_{\delta>0} \overline{H_{\eta(\delta)}(U)} .
$$

Let $x \in H_{\mathrm{co}}\left(x_{0}, b\right)$. Let us show that $x$ is a limit point of the set $H_{\eta(\delta)}(U)$ for any $\delta>0$. By Theorem 4.22, $x$ is a generalized quasisolution of the problem (4.1). Moreover, $x \in U$. Since the set $U$ has Property $\Phi$, we see that there exists a sequence of absolutely continuous functions $x_{i}:[a, b] \rightarrow \mathbb{R}^{n}, i=1,2, \ldots$, such that the following conditions hold: $x_{i} \rightarrow x$ in $\mathbf{C}^{n}[a, b]$ as $i \rightarrow \infty ; x_{i} \in U, \dot{x}_{i} \in \overline{\operatorname{dec}} \Phi(x)$, and $x_{i}(a)=x_{0}$ for each $i=1,2, \ldots$ Suppose that $\eta(\cdot, \cdot) \in$ $K([a, b] \times[0, \infty))$ provides a uniform (in the sense of Definition 5.6) estimate from above for the modulus of continuity of the mapping $\Delta:[a, b] \times \mathbf{C}^{n}[a, b] \rightarrow \operatorname{comp}\left[\mathbb{R}^{n}\right]$. Then there exists $i_{1}$ such that $\left\|x-x_{i}\right\|_{\mathrm{C}^{n}[a, b]}<\omega(x, \delta)$ for each $i \geqslant i_{1}$. This implies that $x_{i} \in \overline{B_{\mathrm{C}^{n}[a, b]}[x, \omega(x, \delta)]}$ for each $i \geqslant i_{1}$. Therefore, $x_{i} \in M_{U}(\omega)(x, \delta)$ for each $i \geqslant i_{1}$. By Definition 5.6, there exists a number $i_{2} \geqslant i_{1}$ such that

$$
\varphi_{U}(\omega)\left(t, x,\left\|x-x_{i}\right\|_{\mathrm{C}^{n}[a, b]}\right) \leqslant \eta(t, \delta)
$$

for any $i \geqslant i_{2}$ and almost all $t \in[a, b]$.

The inequality (5.19) yields that

$$
\rho\left[\dot{x}_{i}(t) ; \Delta\left(t, x_{i}\right)\right] \leqslant h\left[\Delta(t, x) ; \Delta\left(t, x_{i}\right)\right] \leqslant \varphi_{U}(\omega)\left(t, x,\left\|x-x_{i}\right\|_{\mathbf{C}^{n}[a, b]}\right) \leqslant \eta(t, \delta)
$$

for each $i \geqslant i_{2}$ and almost all $t \in[a, b]$. By (5.20), $x_{i} \in H_{\eta(\delta)}(U)$ for each $i \geqslant i_{2}$. This implies that $x$ is a limit point of the set $H_{\eta(\delta)}(U)$. Therefore, $x \in \overline{H_{\eta(\delta)}(U)}$, and consequently (5.18), is satisfied. 
Let us prove the opposite inclusion

$$
\bigcap_{\delta>0} \overline{H_{\eta(\delta)}(U)} \subset H_{\mathrm{co}}\left(x_{0}, b\right)
$$

Let $x \in \bigcap_{\delta>0} \overline{H_{\eta(\delta)}(U)}$. This implies that for each $i=1,2, \ldots$, there exists $x_{i} \in H_{\eta(1 / i)}(U)$ satisfying $\left\|x-x_{i}\right\|_{\mathrm{C}^{n}[a, b]}<1 / i$. Suppose that functions $z_{i} \in \overline{\operatorname{dec}} \Phi(x)$ satisfy

$$
\left|\dot{x}_{i}(t)-z_{i}(t)\right|=\rho\left[\dot{x}_{i}(t) ; \Delta(t, x)\right]
$$

for each $i=1,2, \ldots$ and almost all $t \in[a, b]$. Let us show that

$$
\lim _{i \rightarrow \infty}\left\|\dot{x}_{i}-z_{i}\right\|_{L_{1}^{n}[a, b]}=0 .
$$

Since $\eta(\cdot, \cdot) \in K([a, b] \times[0, \infty))$, by the Lebesgue theorem, we have that

$$
\lim _{i \rightarrow \infty} \int_{a}^{b} \eta\left(t, \frac{1}{i}\right) d t=0
$$

By (5.22), the estimates

$$
\left|\dot{x}_{i}(t)-z_{i}(t)\right| \leqslant h\left[\Delta\left(t, x_{i}\right)^{\eta(t, 1 / i)} ; \Delta(t, x)\right] \leqslant \eta\left(t, \frac{1}{i}\right)+h\left[\Delta(t, x) ; \Delta\left(t, x_{i}\right)\right]
$$

are satisfied for each $i=1,2, \ldots$ and almost all $t \in[a, b]$. Therefore,

$$
\int_{a}^{b}\left|\dot{x}_{i}(t)-z_{i}(t)\right| d t \leqslant \int_{a}^{b} \eta\left(t, \frac{1}{i}\right) d t+2 h_{\mathbf{L}_{1}^{n}[a, b]}\left[\overline{\operatorname{dec}} \Phi(x) ; \overline{\operatorname{dec}} \Phi\left(x_{i}\right)\right]
$$

for each $i=1,2, \ldots$ By (5.24) and due to the continuity of the mapping $\widetilde{\Phi}: \mathbf{C}^{n}[a, b] \rightarrow$ $\Pi\left[\mathbf{L}_{1}^{n}[a, b]\right]$ given by (3.25), we have (5.23).

Since $\dot{x}_{i} \rightarrow \dot{x}$ weakly in $\mathbf{L}_{1}^{n}[a, b]$ as $i \rightarrow \infty$, we have that $z_{i} \rightarrow \dot{x}$ weakly in $\mathbf{L}_{1}^{n}[a, b]$ as $i \rightarrow \infty$. Therefore, by [21], $\dot{x} \in \widetilde{\Phi}_{\mathrm{co}}(x)$ and hence $x \in H\left(x_{0}, b\right)$. Thus, (5.21) is valid. Hence, (5.17) holds and the proof is complete.

Theorem 5.10. Suppose that a set $U \subset \mathrm{C}^{n}[a, b]$ has Property $\boldsymbol{9}$. Then,

$$
\overline{H\left(x_{0}, b\right)}=\bigcap_{\delta>0} \overline{H_{\eta(\delta)}(U)}
$$

for any $\eta(\cdot, \cdot) \in K([a, b] \times[0, \infty))$ if and only if the equality $(4.51)$ is satisfied.

Proof. Let us prove the sufficiency. Assume that (4.51) holds. Let us show that the equality (5.27) is satisfied for any function $\eta(\cdot, \cdot) \in K([a, b] \times[0, \infty))$. By the definition of the problem (5.16), the inclusion

$$
H\left(x_{0}, b\right) \subset H_{\eta(\delta)}(U)
$$


is satisfied for any $\delta>0$. Therefore, for any $\delta>0$, we have the inclusion

$$
\overline{H\left(x_{0}, b\right)} \subset \overline{H_{\eta(\delta)}(U)}
$$

and consequently the inclusion

$$
\overline{H\left(x_{0}, b\right)} \subset \bigcap_{\delta>0} \overline{H_{\eta(\delta)}(U)}
$$

holds. Now, let us check that the opposite relation

$$
\bigcap_{\delta>0} \overline{H_{\eta(\delta)}(U)} \subset \overline{H\left(x_{0}, b\right)},
$$

which is, by (4.51), equivalent to the inclusion

$$
\bigcap_{\delta>0} \overline{H_{\eta(\delta)}(U)} \subset H_{\mathrm{co}}\left(x_{0}, b\right),
$$

holds as well. The latter relation can be proven similarly to Theorem 5.9.

The necessity follows readily from Theorem 5.9. The proof is complete.

Remark 5.11. Note that the equality (5.27) describes the robustness property of the set $H\left(x_{0}, b\right)$ with respect to external perturbations $\eta(\cdot, \cdot) \in K([a, b] \times[0, \infty))$. These external perturbations (e.g., $\eta(\cdot, \cdot) \in K([a, b] \times[0, \infty)))$ characterize an error in measurements of the values of the mapping $\widetilde{\Phi}: \mathbf{C}^{n}[a, b] \rightarrow \Pi\left[\mathbf{L}_{1}^{n}[a, b]\right]$ given by (3.25).

On the other hand, each generalized solution $x:[a, b] \rightarrow \mathbb{R}^{n}$ of the problem (4.1) may also be measured with a certain error. This error may be described by a function belonging to the set $P\left(\mathrm{C}^{n}[a, b] \times[0, \infty)\right)$ and it may be characterized by so-called internal perturbations, which are defined below. Let us show further that internal perturbations influence essentially the properties of generalized solutions of the problem (4.1).

We define a mapping $\Delta_{\text {ext }}:[a, b] \times \mathbf{C}^{n}[a, b] \rightarrow \operatorname{comp}\left[\mathbb{R}^{n}\right]$ by

$$
\Delta_{\text {ext }}(t, x)=\overline{\operatorname{ext}}(\cos \Delta(t, x)),
$$

where $\Delta:[a, b] \times \mathrm{C}^{n}[a, b] \rightarrow \operatorname{comp}\left[\mathbb{R}^{n}\right]$ is the mapping generating the operator $\widetilde{\Phi}: \mathbf{C}^{n}[a, b] \rightarrow \Pi\left[\mathbf{L}_{1}^{n}[a, b]\right]$ given by (3.25); see the definition of $\overline{\operatorname{ext}}(\operatorname{co} \Delta(t, x))$ in Section 2. Let us remark that the mapping $\Delta_{\text {ext }}(\cdot, x)$ is measurable (see [25]) and integrally bounded for each $x \in \mathbf{C}^{n}[a, b]$.

Consider the operator $\widetilde{\Phi}_{\text {ext }}: \mathbf{C}^{n}[a, b] \rightarrow \Pi\left[\mathbf{L}_{1}^{n}[a, b]\right]$ given by

$$
\widetilde{\Phi}_{\mathrm{ext}}(x)=S\left(\Delta_{\mathrm{ext}}(\cdot, x)\right),
$$

where the mapping $\Delta_{\text {ext }}:[a, b] \times C^{n}[a, b] \rightarrow \operatorname{comp}\left[\mathbb{R}^{n}\right]$ is given by (5.33).

Remark 5.12. Note that for each $x \in \mathbf{C}^{n}[a, b]$, the set $\widetilde{\Phi}_{\text {ext }}(x)$ has the following property

$$
\overline{\mathrm{CO}}\left(\tilde{\Phi}_{\mathrm{ext}}(x)\right)=\overline{\mathrm{CO}}(\overline{\operatorname{dec}} \Phi(x)) .
$$

Also, $\widetilde{\Phi}_{\text {ext }}(x)$ is the minimal set among all nonempty closed in $\mathbf{L}_{1}^{n}[a, b]$ decomposable subsets of $\overline{\operatorname{dec}} \Phi(x)$ satisfying (5.35). 
Consider the problem

$$
\dot{x} \in \widetilde{\Phi}_{\mathrm{ext}}(x), \quad x(a)=x_{0} \quad\left(x_{0} \in \mathbb{R}^{n}\right) .
$$

We call any solution (resp., quasisolution) of (5.36) a generalized extreme (in the sense of the definition in Section 2) solution (resp., generalized extreme quasisolution) of the problem (4.1).

Let $\mathscr{t}_{\text {ext }}\left(x_{0}\right)$ be the set of all generalized extreme quasisolutions of the problem (4.1). Theorem 4.22, Remark 4.23, and equality (5.35) imply the following result.

Corollary 5.13. $\mathscr{L}_{\mathrm{ext}}\left(x_{0}\right)=H_{\mathrm{co}}\left(x_{0}, b\right)$.

Let $\eta(\cdot, \cdot) \in K([a, b] \times[0, \infty)), \omega(\cdot, \cdot) \in P\left(\mathbf{C}^{n}[a, b] \times[0, \infty)\right)$. Let also $U$ be a convex closed set in $\mathbf{C}^{n}[a, b]$. We define mappings $\widetilde{\Phi}_{\eta, \omega}: U \times[0, \infty) \rightarrow \operatorname{comp}\left[\mathbf{L}_{1}^{n}[a, b]^{*}\right], \Delta_{\mathrm{ext}, \eta}:[a, b] \times$ $\mathbf{C}^{n}[a, b] \times[0, \infty) \rightarrow \operatorname{comp}\left[\mathbb{R}^{n}\right], \tilde{\Phi}_{\mathrm{ext}, \eta}: \mathbf{C}^{n}[a, b] \times[0, \infty) \rightarrow \Pi\left[\mathbf{L}_{1}^{n}[a, b]\right], \widetilde{\Phi}_{\mathrm{ext}, \eta, \omega}: \mathbf{C}^{n}[a, b] \times$ $[0, \infty) \rightarrow \operatorname{comp}\left[\mathbf{L}_{1}^{n}[a, b]^{*}\right]$ by the formulas

$$
\begin{gathered}
\widetilde{\Phi}_{\eta, \omega}(x, \delta)=\widetilde{\Phi}_{\eta}\left(\left(M_{U}(\omega)(x, \delta)\right), \delta\right), \\
\left(\Delta_{\mathrm{ext}, \eta}\right)(t, x, \delta)=\left(\Delta_{\mathrm{ext}}(t, x)\right)^{\eta(t, \delta),} \\
\left(\widetilde{\Phi}_{\mathrm{ext}, \eta}\right)(x, \delta)=S\left(\Delta_{\mathrm{ext}, \eta}(\cdot, x, \delta)\right), \\
\widetilde{\Phi}_{\mathrm{ext}, \eta, \omega}(x, \delta)=\widetilde{\Phi}_{\mathrm{ext}, \eta}\left(\left(M_{U}(\omega)(x, \delta)\right), \delta\right),
\end{gathered}
$$

where the mappings $\widetilde{\Phi}_{\eta}: \mathbf{C}^{n}[a, b] \times[0, \infty) \rightarrow \Pi\left[\mathbf{L}_{1}^{n}[a, b]\right], M_{U}(\omega): U \times[0, \infty) \rightarrow \Omega(U)$, $\Delta_{\text {ext }}:[a, b] \times \mathbf{C}^{n}[a, b] \times[0, \infty) \rightarrow \operatorname{comp}\left[\mathbb{R}^{n}\right]$ are given by the equalities (5.5), (5.9), and (5.33), respectively.

For each $\delta>0$, consider the following problems on $U \subset \mathbf{C}^{n}[a, b]$ :

$$
\begin{gathered}
\dot{x} \in \widetilde{\Phi}_{\eta, \omega}(x, \delta), \quad x(a)=x_{0} \quad\left(x_{0} \in \mathbb{R}^{n}\right), \\
\dot{x} \in \widetilde{\Phi}_{\mathrm{ext}, \eta, \omega}(x, \delta), \quad x(a)=x_{0} \quad\left(x_{0} \in \mathbb{R}^{n}\right),
\end{gathered}
$$

where the mappings $\widetilde{\Phi}_{\eta, \omega}: U \times[0, \infty) \rightarrow \operatorname{comp}\left[\mathbf{L}_{1}^{n}[a, b]^{*}\right], \widetilde{\Phi}_{\mathrm{ext}, \eta, \omega}: U \times[0, \infty) \rightarrow \operatorname{comp}\left[\mathbf{L}_{1}^{n}[a, b]^{*}\right]$ are given by (5.37).

We call any solution of the problem (5.38) with a fixed $\delta>0$ a generalized $\delta$-solution of the problem (4.1), or a generalized approximate solution of (4.1) with external and internal perturbations. For each $\delta>0$, we denote by $H_{\eta(\delta), \omega(\delta)}(U)\left(H_{\mathrm{ext}, \eta(\delta), \omega(\delta)}(U)\right)$ the set of all solutions of the problem (5.38) ((5.39)) on $U \subset \mathbf{C}^{n}[a, b]$. Since $\widetilde{\Phi}_{\mathrm{ext}, \eta, \omega}(x, \delta) \subset \widetilde{\Phi}_{\eta, \omega}(x, \delta)$ for any $\delta>0$ and any $x \in U$, we see that $H_{\mathrm{ext}, \eta(\delta), \omega(\delta)}(U) \subset H_{\eta(\delta), \omega(\delta)}(U)$ for any $\delta>0$.

Theorem 5.14. Let the set $U \subset \mathbf{C}^{n}[a, b]$ have Property $\Phi$. Then for any $\eta(\cdot, \cdot) \in K([a, b] \times[0, \infty))$, $\omega(\cdot, \cdot) \in P\left(\mathbf{C}^{n}[a, b] \times[0, \infty)\right)$, one has

$$
H_{\mathrm{co}}\left(x_{0}, b\right)=\bigcap_{\delta>0} \overline{H_{\mathrm{ext}, \eta(\delta), \omega(\delta)}(U)}=\bigcap_{\delta>0} \overline{H_{\eta(\delta), \omega(\delta)}(U)},
$$

where $\overline{H_{\mathrm{ext}, \eta(\delta), \omega(\delta)}(U)}$ and $\overline{H_{\eta(\delta), \omega(\delta)}(U)}$ are the closures of the sets $H_{\mathrm{ext}, \eta(\delta), \omega(\delta)}(U)$ and $H_{\eta(\delta), \omega(\delta)}(U)$, respectively, in the space $\mathbf{C}^{n}[a, b]$. 
Proof. First of all, let us check that

$$
H_{\mathrm{co}}\left(x_{0}, b\right) \subset \bigcap_{\delta>0} \overline{H_{\mathrm{ext}, \eta(\delta), \omega(\delta)}(U)}
$$

Let $x \in H\left(x_{0}, b\right)$. We show that $x$ is a limit point of the set $H_{\mathrm{ext}, \eta(\delta), \omega(\delta)}(U)$ for any $\delta>0$. By Corollary 5.13, $x$ is a generalized extreme quasisolution of the problem (4.1). Since the set $U$ has Property $\Phi$, we see that $x \in U$ and there exists a sequence of absolutely continuous functions $x_{i}:[a, b] \rightarrow \mathbb{R}^{n}, i=1,2, \ldots$, with the following properties: $x_{i} \rightarrow x$ in $\mathbf{C}^{n}[a, b]$ as $i \rightarrow \infty ; x_{i} \in U$, $\dot{x}_{i} \in \widetilde{\Phi}_{\text {ext }}(x)$, and $x_{i}(a)=x_{0}$ for each $i=1,2, \ldots$. Here, the operator $\widetilde{\Phi}_{\text {ext }}: \mathbf{C}^{n}[a, b] \rightarrow \Pi\left[\mathbf{L}_{1}^{n}[a, b]\right]$ is given by (5.33) and (5.34).

Let us prove also that there exists a number $i_{0}$ such that for each $i \geqslant i_{0}$,

$$
x_{i} \in H_{\mathrm{ext}, \eta(\delta), \omega(\delta)}(U)
$$

Since $\omega(\cdot, \cdot) \in P\left(\mathbf{C}^{n}[a, b] \times[0, \infty)\right)$, we see that there exists a number $i_{0}$ such that for each $i \geqslant i_{0}$, $x \in B_{\mathrm{C}^{n}[a, b]}\left[x_{i} ; \omega\left(x_{i}, \delta\right)\right]$. This implies that for each $i \geqslant i_{0}$, we have that $x \in M_{U}\left(x_{i}, \delta\right)$ (see (5.9)). Therefore, for each $i \geqslant i_{0}$, the inclusion $\widetilde{\Phi}_{\mathrm{ext}}(x) \subset \widetilde{\Phi}_{\mathrm{ext}, \eta, \omega}\left(x_{i}, \delta\right)$ holds. Hence, for each $i \geqslant i_{0}$, we have (5.42). This means that $x$ is a limit point of the set $H_{\text {ext, } \eta(\delta), \omega(\delta)}(U)$. Therefore, $x \in \overline{H_{\mathrm{ext}, \eta(\delta), \omega(\delta)}(U)}$ and (5.41) is satisfied.

The relation

$$
\bigcap_{\delta>0} \overline{H_{\mathrm{ext}, \eta(\delta), \omega(\delta)}(U)} \subset H_{\mathrm{co}}\left(x_{0}, b\right)
$$

can be proven similarly to (5.21) (see the proof of Theorem 5.9). The second equality of (5.40) can be proven in the same way. This completes the proof of the theorem.

Remark 5.15. Theorem 5.14 says that no measurement accuracy of the values of the mapping $\Delta:[a, b] \times \mathbf{C}^{n}[a, b] \rightarrow \operatorname{comp}\left[\mathbb{R}^{n}\right]$ could guarantee the "reconstruction" of the set $\overline{H\left(x_{0}, b\right)}$ by means of $\overline{H_{\eta(\delta), \omega(\delta)}(U)}$. That is only possible if the density principle holds for the generalized solutions.

\section{Conclusion}

The main results of the paper can be summarized as follows. For the decomposable hull of a mapping, we have obtained the conditions for the property of the Hausdorff lower semicontinuity (resp., upper semicontinuity and continuity). We considered a functional differential inclusion with a Volterra multivalued mapping which is not necessarily decomposable. The concept of a generalized solution of the initial value problem for such an inclusion was introduced and its properties were studied. Conditions for the local existence and continuation of a generalized solution to the initial value problem were obtained. We have offered some estimates, which characterize the closeness of generalized solutions and a given absolutely continuous function. These estimates were derived from the conditions for the existence of a generalized solution satisfying the inequality (4.18) (see Theorem 4.13 and Corollary 4.14).

The concept of a generalized quasisolution of the initial value problem was introduced. We proved that the set of all generalized quasisolutions of the initial value problem coincides 
with the solution set of the functional differential inclusion with the convex decomposable hull of the right-hand side. Using this fact as well as the estimates characterizing the closeness of generalized solutions and a given absolutely continuous function, we obtained the density principle for the generalized solutions.

Asymptotic properties of the set of generalized approximate solutions (generalized $\delta$ solutions) were studied. It was proven that the limit of the closures of the sets of generalized approximate solutions coincides with the closure of the set of the generalized solutions if and only if the density principle holds for the generalized solutions.

\section{Acknowledgments}

The first author is supported by CIGENE (Center for Integrative Genetics) and by Lånekassen (Norwegian State Educational Loan Fund). The second author is supported by CIGENE and partially supported by the Russian FBR Grant no. 07-01-00305. The third author is partially supported by the Russian FBR Grant no. 07-01-00305.

\section{References}

[1] A. I. Bulgakov and L. I. Tkach, "Perturbation of a convex-valued operator by a Hammersteintype multivalued mapping with nonconvex images, and boundary value problems for functionaldifferential inclusions," Matematicheskiŭ Sbornik, vol. 189, no. 6, pp. 3-32, 1998, English translation in Sbornik. Mathematics, vol. 189, no. 5-6, pp. 821-848, 1998.

[2] A. I. Bulgakov and L. I. Tkach, "Perturbation of a single-valued operator by a multi-valued mapping of Hammerstein type with nonconvex images," Izvestiya Vysshikh Uchebnykh Zavedenit. Matematika, no. 3, pp. 3-16, 1999, English translation in Russian Mathematics, vol. 43, no. 3, pp. 1-13, 1999.

[3] A. F. Filippov, "Classical solutions of differential equations with the right-hand side multi-valued," Vestnik Moskovskogo Universiteta. Serija I. Matematika, Mehanika, vol. 22, no. 3, pp. 16-26, 1967 (Russian).

[4] A. F. Filippov, Differential Equations with Discontinuous Right-Hand Sides, Nauka, Moscow, Russia, 1985.

[5] A. I. Bulgakov, "Asymptotic representation of sets of $\delta$-solutions of a differential inclusion," Matematicheskie Zametki, vol. 65, no. 5, pp. 775-779, 1999, English translation in Mathematical Notes, vol. 65, no. 5-6, pp. 649-653, 1999.

[6] A. I. Bulgakov, O. P. Belyaeva, and A. A. Grigorenko, "On the theory of perturbed inclusions and its applications," Matematicheskiu Sbornik, vol. 196, no. 10, pp. 21-78, 2005, English translation in Sbornik. Mathematics, vol. 196, no. 9-10, pp. 1421-1472, 2005.

[7] A. I. Bulgakov, A. A. Efremov, and E. A. Panasenko, "Ordinary differential inclusions with internal and external perturbations," Differentsial'nye Uravneniya, vol. 36, no. 12, pp. 1587-1598, 2000, English translation in Differential Equations, vol. 36, no. 12, pp. 1741-1753, 2000.

[8] A. I. Bulgakov and V. V. Skomorokhov, "Approximation of differential inclusions," Matematicheskiu Sbornik, vol. 193, no. 2, pp. 35-52, 2002, English translation in Sbornik. Mathematics, vol. 193, no. 1-2, pp. 187-203, 2002.

[9] T. Ważewski, "Sur une généralisation de la notion des solutions d'une équation au contingent," Bulletin de l'Académie Polonaise des Sciences. Série des Sciences Mathématiques, Astronomiques et Physiques, vol. 10, pp. 11-15, 1962.

[10] V. I. Blagodatskikh and A. F. Filippov, "Differential inclusions and optimal control," Trudy Matematicheskogo Instituta Imeni V. A. Steklova, vol. 169, pp. 194-252, 1985, English translation in Proceedings of the Steklov Institute of Mathematics, vol. 169, 1986.

[11] A. Bressan, "On a bang-bang principle for nonlinear systems," Bollettino della Unione Matemàtica Italiana. Supplemento, no. 1, pp. 53-59, 1980.

[12] A. E. Irisov and E. L. Tonkov, "On the closure of the set of periodic solutions of a differential inclusion," in Differential and Integral Equations, pp. 32-38, Gor' kov. Gos. Univ., Gorki, Russia, 1983.

[13] G. Pianigiani, "On the fundamental theory of multivalued differential equations," Journal of Differential Equations, vol. 25, no. 1, pp. 30-38, 1977. 
[14] L. N. Lyapin and Yu. L. Muromtsev, "Guaranteed optimal control on a set of operative states," $A u$ tomation and Remote Control, vol. 54, no. 3, part 1, pp. 421-429, 1993 (Russian).

[15] M. S. Branicky, V. S. Borkar, and S. K. Mitter, "A unified framework for hybrid control: model and optimal control theory," IEEE Transactions on Automatic Control, vol. 43, no. 1, pp. 31-45, 1998.

[16] R. W. Brockett, "Hybrid models for motion control systems," in Essays on Control: Perspectives in the Theory and Its Applications (Groningen, 1993), H. Trentelman and J. C. Willems, Eds., vol. 14 of Progress in Systems Control Theory, pp. 29-53, Birkhäuser, Boston, Mass, USA, 1993.

[17] J. Lygeros, C. Tomlin, and S. Sastry, "Controllers for reachability specifications for hybrid systems," Automatica, vol. 35, no. 3, pp. 349-370, 1999.

[18] A. Puri and P. Varaiya, "Decidability of hybrid systems with rectangular differential inclusions," in Computer Aided Verification (Stanford, CA, 1994), D. Dill, Ed., vol. 1066 of Lecture Notes in Computer Science, pp. 95-104, Springer, Berlin, Germany, 1994.

[19] A. J. Van der Schaft and J. M. Schumacher, An Introduction to Hybrid Dynamical Systems, vol. 251 of Springer Lecture Notes in Control and Information Sciences, Springer, London, UK, 2000.

[20] P. Varaiya and A. Kurzhanski, "On problems of dynamics and control for hybrid systems," in Control Theory and Theory of Generalized Solutions of Hamilton Jacobi Equations. Proceedings of International Seminars, vol. 1, pp. 21-37, Ural University, Ekaterinburg, Russia, 2006.

[21] A. D. Ioffe and V. M. Tikhomirov, Theory of External Problems, Nauka, Moscow, Russia, 1974.

[22] I. P. Natanson, Theory of Functions of a Real Variable, Nauka, Moscow, Russia, 3rd edition, 1974.

[23] J.-P. Aubin and A. Cellina, Differential Inclusions: Set-Valued Maps and Viability Theory, vol. 264, Springer, Berlin, Germany, 1984.

[24] M. Kamenskii, V. Obukhovskii, and P. Zecca, Condensing Multivalued Maps and Semilinear Differential Inclusions in Banach Spaces, vol. 7 of de Gruyter Series in Nonlinear Analysis and Applications, Walter de Gruyter, Berlin, Germany, 2001.

[25] A. A. Tolstonogov, Differential Inclusions in a Banach Space, Nauka, Novosibirsk, Russia, 1986.

[26] A. N. Tikhonov, "On Volterra type functional equations and their applications in some problems of mathematical physics," Bulletin of Moscow University, Section A, vol. 1, no. 8, pp. 1-25, 1938 (Russian).

[27] A. Bressan and G. Colombo, "Extensions and selections of maps with decomposable values," Studia Mathematica, vol. 90, no. 1, pp. 69-86, 1988.

[28] A. Fryszkowski, "Continuous selections for a class of nonconvex multivalued maps," Studia Mathematica, vol. 76, no. 2, pp. 163-174, 1983.

[29] A. I. Bulgakov, "A functional-differential inclusion with an operator that has nonconvex images," Differentsial'nye Uravneniya, vol. 23, no. 10, pp. 1659-1668, 1987, English translation in Differential Equations, vol. 23, 1987.

[30] A. I. Bulgakov, "Continuous branches of multivalued functions, integral inclusions with nonconvex images, and their applications. I," Differentsial'nye Uravneniya, vol. 28, no. 3, pp. 371-379, 1992.

[31] A. I. Bulgakov, "Continuous branches of multivalued functions, integral inclusions with nonconvex images, and their applications. II," Differentsial'nye Uravneniya, vol. 28, pp. 566-571, 1992.

[32] A. I. Bulgakov, "Continuous branches of multivalued mappings, and integral inclusions with nonconvex images and their applications. III," Differentsial'nye Uravneniya, vol. 28, no. 5, pp. 739-746, 1992.

[33] A. Turowicz, "Remarque sur la définition des quasitrajectoires d'un système de commande nonlinéaire," Bulletin de l'Académie Polonaise des Sciences. Série des Sciences Mathématiques, Astronomiques et Physiques, vol. 11, pp. 367-368, 1963.

[34] A. Pliś, "Trajectories and quasitrajectories of an orientor field," Bulletin de l'Académie Polonaise des Sciences. Série des Sciences Mathématiques, Astronomiques et Physiques, vol. 11, pp. 369-370, 1963.

[35] A. I. Bulgakov, "Integral inclusions with nonconvex images and their applications to boundary value problems for differential inclusions," Matematicheskiu Sbornik, vol. 183, no. 10, pp. 63-86, 1992, English translation in Russian Academy of Sciences. Sbornik. Mathematics, vol. 77, no. 1, pp. 193-212, 1994.

[36] A. I. Bulgakov and V. P. Maksimov, "Functional and functional-differential inclusions with Volterra operators," Differential Equations, vol. 17, no. 8, pp. 881-890, 1981.

[37] A. V. Arutyunov, Optimality Conditions: Abnormal and Degenerate Problems, vol. 526 of Mathematics and Its Applications, Kluwer Academic Publishers, Dordrecht, The Netherlands, 2000.

[38] A. A. Tolstonogov and P. I. Chugunov, "The solution set of a differential inclusion in a Banach space. I," Sibirskĭ Matematicheskiŭ Zhurnal, vol. 24, no. 6, pp. 144-159, 1983, English translation in Siberian Mathematical Journal, vol. 24, no. 6, pp. 941-954, 1983. 
[39] A. A. Tolstonogov and I. A. Finogenko, "Solutions of a differential inclusion with lower semicontinuous nonconvex right-hand side in a Banach space," Matematicheskiu Sbornik, vol. 125(167), no. 2, pp. 199-230, 1984, English translation in Sbornik. Mathematics, vol. 53, no. 1, pp. 203-231, 1986.

[40] O. Hájek, “Discontinuous differential equations. I," Journal of Differential Equations, vol. 32, no. 2, pp. 149-170, 1979.

[41] O. Hájek, "Discontinuous differential equations. II," Journal of Differential Equations, vol. 32, no. 2, pp. 171-185, 1979.

[42] H. Hermes, "The generalized differential equation $x \in R(t, x)$, " Advances in Mathematics, vol. 4, pp. $149-169,1970$.

[43] H. Hermes, "On continuous and measurable selections and the existence of solutions of generalized differential equations," Proceedings of the American Mathematical Society, vol. 29, pp. 535-542, 1971. 


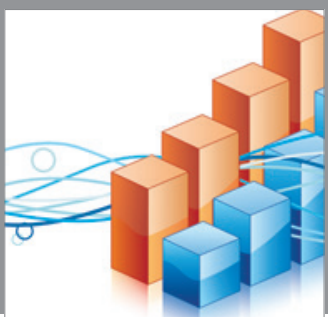

Advances in

Operations Research

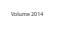

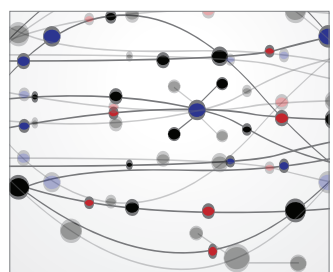

\section{The Scientific} World Journal
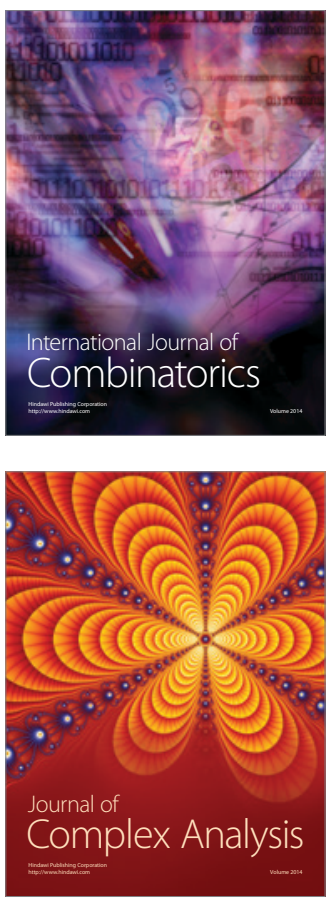

International Journal of

Mathematics and

Mathematical

Sciences
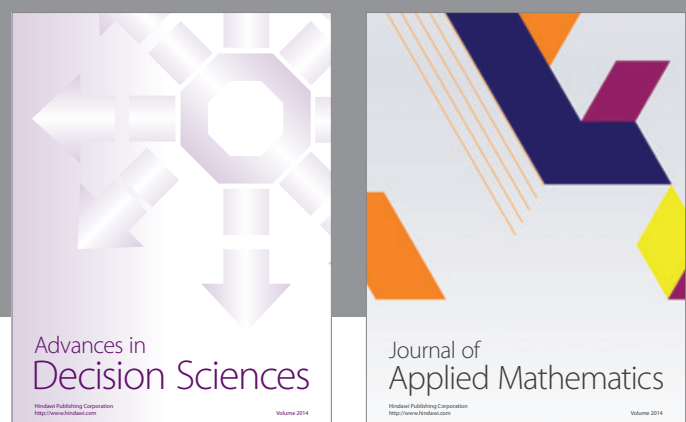

Journal of

Applied Mathematics
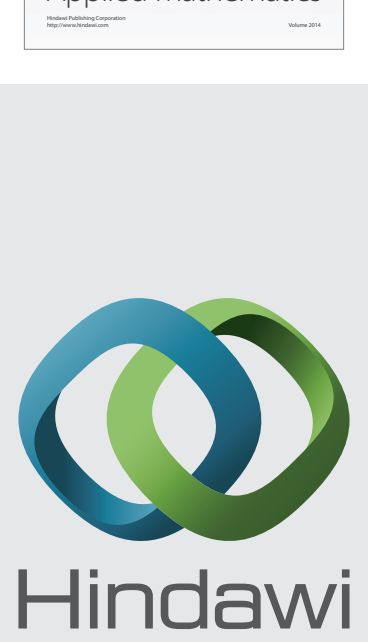

Submit your manuscripts at http://www.hindawi.com
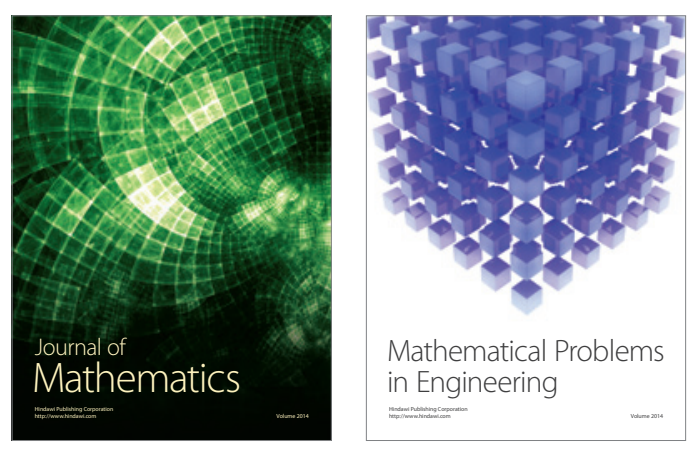

Mathematical Problems in Engineering
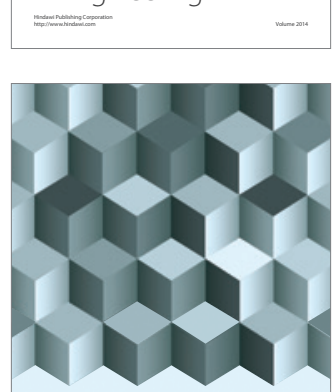

Journal of

Function Spaces
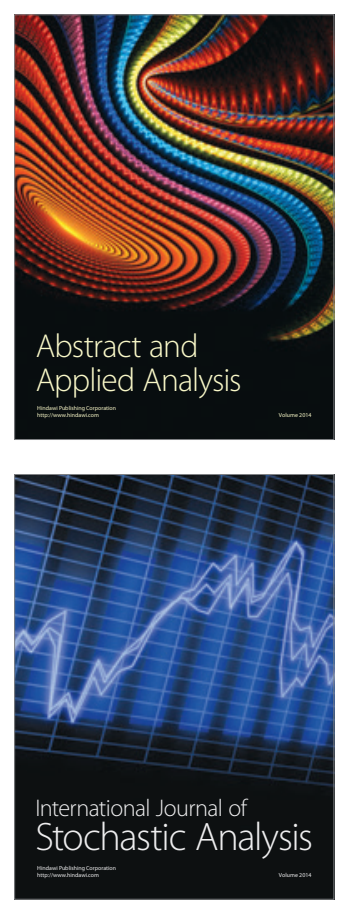

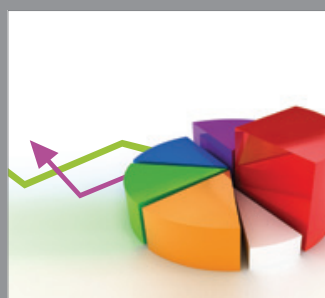

ournal of

Probability and Statistics

Promensencen
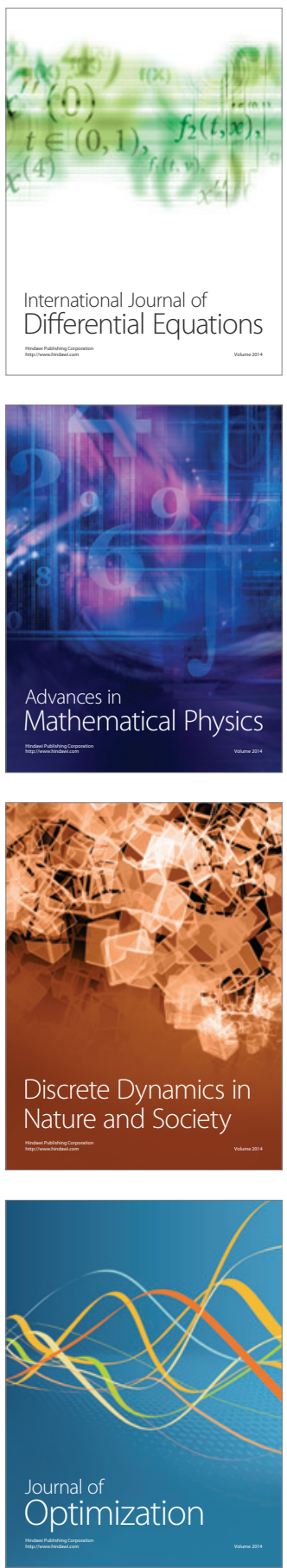